JOURNAL OF THE AMERICAN MATHEMATICAL SOCIETY

Volume 20, Number 4, October 2007, Pages 1111-1133

S 0894-0347(07)00562-0

Article electronically published on April 23, 2007

\title{
TROPICAL DISCRIMINANTS
}

\author{
ALICIA DICKENSTEIN, EVA MARIA FEICHTNER, AND BERND STURMFELS
}

Dedicated to the memory of Pilar Pisón Casares

\section{INTRODUCTION}

Let $A$ be an integer $d \times n$-matrix such that $(1,1, \ldots, 1)$ is in the row span of $A$. This defines a projective toric variety $X_{A}$ in $\mathbb{C P}^{n-1}$. Its dual variety $X_{A}^{*}$ is the closure in the projective space dual to $\mathbb{C P}^{n-1}$ of the set of hyperplanes that are tangent to $X_{A}$ at a regular point. The toric variety $X_{A}$ is called non-defective if its dual variety $X_{A}^{*}$ has codimension one. In this case, the $A$-discriminant is the irreducible homogeneous polynomial $\Delta_{A}$ which defines the hypersurface $X_{A}^{*}$. Alternatively, the dual variety $X_{A}^{*}$ can be thought of as the set of singular hypersurfaces in $\left(\mathbb{C}^{*}\right)^{d}$ with Newton polytope prescribed by the matrix $A$. The study of these objects is an active area of research in computational algebraic geometry, with the fundamental reference being the monograph by Gel'fand, Kapranov and Zelevinsky [13].

Our main object of interest in this paper is the tropical A-discriminant $\tau\left(X_{A}^{*}\right)$. This is the polyhedral fan in $\mathbb{R}^{n}$ which is obtained by tropicalizing $X_{A}^{*}$. While it is generally difficult to compute the dual variety $X_{A}^{*}$ from $A$, we show that its tropicalization $\tau\left(X_{A}^{*}\right)$ can be computed much more easily. In Theorem 1.1, we derive an explicit description of the tropical $A$-discriminant $\tau\left(X_{A}^{*}\right)$, and in Theorem 1.2 we present an explicit combinatorial formula for the extreme monomials of $\Delta_{A}$.

Without loss of generality, we assume that the columns of the matrix $A$ span the integer lattice $\mathbb{Z}^{d}$ and that the point configuration given by the columns of $A$ is not a pyramid. These hypotheses ensure that the toric variety $X_{A}$ has dimension $d-1$ and that the dual variety $X_{A}^{*}$ is not contained in any coordinate hyperplane.

A key player in this paper is the tropicalization of the kernel of $A$. As shown in [1] and 10, this tropical linear space is subdivided both by the Bergman fan of the matroid dual to $A$, i.e., the co-Bergman fan $\mathcal{B}^{*}(A)$ of $A$, and by the nested set fans of its lattice of flats $\mathcal{L}(A)$. In other words, let $\mathcal{L}(A)$ denote the geometric lattice whose elements are the sets of zero-entries of the vectors in $\operatorname{kernel}(A)$, ordered by inclusion. We write $\mathcal{C}(A)$ for the set of proper maximal chains in $\mathcal{L}(A)$ and represent these chains as $(n-d-1)$-element subsets $\sigma=\left\{\sigma_{1}, \ldots, \sigma_{n-d-1}\right\}$ of $\{0,1\}^{n}$.

Received by the editors November 8, 2005.

2000 Mathematics Subject Classification. Primary 14M25, Secondary 52B20.

Key words and phrases. Tropical geometry, dual variety, discriminant.

The first author was partially supported by UBACYT X042, CONICET PIP 5617 and ANPCYT 17-20569, Argentina.

The second author was supported by a Research Professorship of the Swiss National Science Foundation, PP002-106403/1.

The last author was partially supported by the U.S. National Science Foundation, DMS0456960.

(c)2007 American Mathematical Society Reverts to public domain 28 years from publication 
We obtain the following descriptions of the tropicalization of the kernel of $A$ :

$$
\tau(\operatorname{kernel}(A))=\operatorname{support}\left(\mathcal{B}^{*}(A)\right)=\bigcup_{\sigma \in \mathcal{C}(A)} \mathbb{R}_{\geq 0} \sigma .
$$

The union on the right hand side in fact is the finest in the hierarchy of unimodular simplicial fan structures provided by the nested set fans [5, 8, 9, 10, 11. The tropical linear space (1.1) is a subset of $\mathbb{R}^{n}$. We obtain the tropical $A$-discriminant by adding this tropical linear space to the (classical) row space of the $d \times n$-matrix $A$ :

Theorem 1.1. For any $d \times n$-matrix $A$ as above, the tropical $A$-discriminant $\tau\left(X_{A}^{*}\right)$ equals the Minkowski sum of the co-Bergman fan $\mathcal{B}^{*}(A)$ and the row space of $A$.

Theorem 1.1 is the tropical analogue of Kapranov's Horn uniformization [16]. By definition, the tropical discriminant $\tau\left(X_{A}^{*}\right)$ inherits the structure of a fan from the Gröbner fan of the ideal of $X_{A}^{*}$ and, in the non-defective case, also from the secondary fan of $A[13$. In general, neither of these two fan structures refines the other, as we shall see in Examples [5.5] and [5.6.

The tropicalization of a variety retains a lot of information about the geometry of the original variety [18, 20, 21, 23, 24.

In Theorem 1.2 below, our tropical approach leads to a formula for the extreme monomials of the $A$-discriminant $\Delta_{A}$, and, a fortiori, for the degree of the dual variety $X_{A}^{*}$. An alternating product formula for the extreme monomials of $\Delta_{A}$ was given in [13, §11.3.C] under the restrictive assumption that $X_{A}$ is smooth. Our formula (1.2) is positive, it is valid for any toric variety $X_{A}$ regardless of smoothness, and its proof is self-contained.

Theorem 1.2. If $X_{A}$ is non-defective and $w$ a generic vector in $\mathbb{R}^{n}$, then the exponent of $x_{i}$ in the initial monomial $\operatorname{in}_{w}\left(\Delta_{A}\right)$ of the A-discriminant $\Delta_{A}$ equals

$$
\sum_{\sigma \in \mathcal{C}_{i, w}}\left|\operatorname{det}\left(A^{t}, \sigma_{1}, \ldots, \sigma_{n-d-1}, e_{i}\right)\right|,
$$

where $\mathcal{C}_{i, w}$ is the subset of $\mathcal{C}(A)$ consisting of all chains such that the row space of the matrix $A$ has non-empty intersection with the cone $\mathbb{R}_{>0}\left\{\sigma_{1}, \ldots, \sigma_{n-d-1},-e_{i},-w\right\}$.

Here, the $A$-discriminant $\Delta_{A}$ is written as a homogeneous polynomial in the variables $x_{1}, \ldots, x_{n}$, and $\operatorname{in}_{w}\left(\Delta_{A}\right)$ is the $w$-lowest monomial $x_{1}^{u_{1}} \cdots x_{n}^{u_{n}}$ which appears in the expansion of $\Delta_{A}$ in characteristic zero. Theorem 1.2 generalizes to the defective case, when we take $\Delta_{A}$ as the Chow form of the dual variety $X_{A}^{*}$. This is stated in Theorem 4.6. Aiming for maximal efficiency in evaluating (1.2) with a computer, we can replace $\mathcal{C}_{i, w}$ with the corresponding maximal nested sets of the geometric lattice $\mathcal{L}(A)$, or with the corresponding maximal cones in the Bergman fan $\mathcal{B}^{*}(A)$. Our maple implementation of the formula (1.2) is discussed in Section 5 .

This paper is organized as follows. In Section 2, we review the construction of the tropicalization $\tau(Y)$ of a projective variety $Y$, and we show how the algebraic cycle underlying any initial monomial ideal of $Y$ can be read off from $\tau(Y)$. In Section 3, we discuss general varieties which are parametrized by a linear map followed by a monomial map. Theorem 3.1 gives a combinatorial description of the tropicalization of the image of such a map. The dual variety $X_{A}^{*}$ of any toric variety $X_{A}$ admits such a parametrization. This is derived in Section 4, and it is used to prove Theorem 1.1 and Theorem 1.2 in the general form of Theorem 4.6. We also obtain in Corollary 4.5 a characterization of the dimension of $X_{A}^{*}$. In 
Section 5 we discuss computational issues, and we examine the connection between the tropical discriminant of $A$ and regular polyhedral subdivisions. In particular, we consider the problem of characterizing $\Delta$-equivalence of regular triangulations in combinatorial terms. Finally, Section 6 is devoted to the case when $A$ is an essential Cayley configuration. The corresponding dual varieties $X_{A}^{*}$ are resultant varieties, and we compute their degrees and initial cycles in terms of mixed subdivisions.

\section{TROPICAL VARIETIES AND THEIR INITIAL CYCLES}

Tropical algebraic geometry is algebraic geometry over the tropical semi-ring $(\mathbb{R} \cup\{\infty\}, \oplus, \odot)$ with arithmetic operations $x \oplus y:=\min \{x, y\}$ and $x \odot y:=x+y$. It transfers the objects of classical algebraic geometry into the combinatorial context of polyhedral geometry. Fundamental references include [7, 18, 19, 20, 21, 24].

Tropicalization is an operation that turns complex projective varieties into polyhedral fans. Let $Y \subset \mathbb{C P}^{n-1}$ be an irreducible projective variety of dimension $r-1$ and let $I_{Y} \subset \mathbb{C}\left[x_{1}, \ldots, x_{n}\right]$ be its homogeneous prime ideal. For $w \in \mathbb{R}^{n}$, we denote by $\operatorname{in}_{w}\left(I_{Y}\right)$ the initial ideal generated by all initial forms $\operatorname{in}_{w}(f)$, for $f \in I_{Y}$, where $\operatorname{in}_{w}(f)$ is the subsum of all terms $c_{u} x^{u}=c_{u} x_{1}^{u_{1}} \cdots x_{n}^{u_{n}}$ in $f$ which have lowest weight $w \cdot u=\sum_{i=1}^{n} w_{i} u_{i}$. The tropicalization $\tau(Y)$ of $Y$ is the set

$$
\tau(Y)=\left\{w \in \mathbb{R}^{n}: \operatorname{in}_{w}\left(I_{Y}\right) \text { does not contain a monomial }\right\} .
$$

Let $K=\mathbb{C}\left\{\left\{\epsilon^{\mathbb{R}}\right\}\right\}$ be the field of Puiseux series, i.e., series with complex coefficients, real exponents and well ordered supports. The elements of $K$ are also known as transfinite Puiseux series, and they form an algebraically closed field of characteristic zero. The order of a non-zero element $z$ in $K^{*}=K \backslash\{0\}$ is the smallest real number $\nu$ such that $\epsilon^{\nu}$ appears with non-zero coefficient in $z$. For a vector $z=\left(z_{1}, \ldots, z_{s}\right)$ in $\left(K^{*}\right)^{s}$ we write $\operatorname{order}(z):=\left(\operatorname{order}\left(z_{1}\right), \ldots, \operatorname{order}\left(z_{s}\right)\right) \in \mathbb{R}^{s}$. The points in the tropicalization $\tau(Y)$ are precisely the orders of $K^{*}$-valued points on the variety $Y$ (see [7, [20, Theorem 2.1.2], 21, Theorem 2.1]).

The set $\tau(Y)$ carries the structure of a polyhedral fan. Namely, it is a subfan of the Gröbner fan of $I_{Y}$; see [23, §9]. By a result of Bieri and Groves [2], the fan $\tau(Y)$ is pure of dimension $r$. In 3 it was shown that $\tau(Y)$ is connected in codimension one, and a practical algorithm was given for computing $\tau(Y)$ from polynomial generators of $I_{Y}$. We will view the tropicalization $\tau(Y)$ of a projective variety as an $(r-1)$-dimensional fan in tropical projective space $\mathbb{P P}^{n-1}:=\mathbb{R}^{n} / \mathbb{R}(1,1, \ldots, 1)$, which is an $(n-1)$-dimensional real affine space.

Every maximal cone $\sigma$ of the fan $\tau(Y)$ comes naturally with an intrinsic multiplicity $m_{\sigma}$, which is a positive integer. The integer $m_{\sigma}$ is computed as the sum of the multiplicities of all monomial-free minimal associated primes of the initial ideal $\operatorname{in}_{w}\left(I_{Y}\right)$ in $\mathbb{C}\left[x_{1}, \ldots, x_{n}\right]$, where $w$ is in the relative interior of the cone $\sigma$.

Remark 2.1. A geometric description of the intrinsic multiplicity $m_{\sigma}$ arises from the beautiful interplay of degenerations and compactifications discovered by Tevelev 24] and studied by Speyer [20, Chapter 2] and Hacking (unpublished). Let $\mathbf{X}$ denote the toric variety associated with the fan $\tau(Y)$. Consider the intersection $Y_{0}$ of $Y$ with the dense torus $T$ in $\mathbb{C P}^{n-1}$, and let $\overline{Y_{0}}$ be the closure of $Y_{0}$ in $\mathbf{X}$. By [24, 1.7, 2.5 , and 2.7], the variety $\overline{Y_{0}}$ is complete and the multiplication map $\Psi: T \times \overline{Y_{0}} \rightarrow \mathbf{X}$ is faithfully flat. If follows that the intersection of $\overline{Y_{0}}$ with a codimension $k$ orbit has codimension $k$ in $\overline{Y_{0}}$. In particular, the orbit $\mathcal{O}(\sigma)$ corresponding to a maximal cone 
$\sigma$ of $\tau(Y)$ intersects $\overline{Y_{0}}$ in a zero-dimensional scheme $Z_{\sigma}$. The intrinsic multiplicity $m_{\sigma}$ of the maximal cone $\sigma$ in the tropical variety $\tau(Y)$ is the length of $Z_{\sigma}$.

We list three fundamental examples which will be important for our work.

(1) Let $Y$ be a hypersurface in $\mathbb{C P}^{n-1}$ defined by an irreducible polynomial $f$ in $\mathbb{C}\left[x_{1}, \ldots, x_{n}\right]$. Then $\tau(Y)$ is the union of all codimension one cones in the normal fan of the Newton polytope of $f$. The intrinsic multiplicity $m_{\sigma}$ of each such cone $\sigma$ is the lattice length of the corresponding edge of the Newton polytope of $f$.

(2) Let $Y=X_{A}$ be the toric variety defined by an integer $d \times n$-matrix $A$ as above. Its tropicalization $\tau\left(X_{A}\right)$ is the linear space spanned by the rows of $A$.

(3) Let $Y$ be a linear subspace in $\mathbb{C}^{n}$ or in $\mathbb{C P}^{n-1}$. The tropicalization $\tau(Y)$ is the Bergman fan of the matroid associated with $Y$; see [1, 10, 23] and (3.2) below.

In the last two families of examples, all the intrinsic multiplicities $m_{\sigma}$ equal 1.

The tropicalization $\tau(Y)$ can be used to compute numerical invariants of $Y$. First, the dimension of $\tau(Y)$ coincides with the dimension of $Y$. In Theorem 2.2 below, we express the multiplicities of the minimal primes in the initial monomial ideals of $I_{Y}$ in terms of $\tau(Y)$. Equivalently, we compute the algebraic cycle of any initial monomial ideal $\operatorname{in}_{w}\left(I_{Y}\right)$. This formula tells us the degree of the variety $Y$, namely, the degree is the sum of the multiplicities of the minimal primes of $\operatorname{in}_{w}\left(I_{Y}\right)$.

Let $c:=n-r$ denote the codimension of the irreducible projective variety $Y$ in $\mathbb{C} \mathbb{P}^{n-1}$. Assume that $Y$ is not contained in a coordinate hyperplane, and let $I_{Y}$ be its homogeneous prime ideal in $\mathbb{C}\left[x_{1}, \ldots, x_{n}\right]$. If $w$ is a generic vector in $\mathbb{R}^{n}$, the initial ideal $\operatorname{in}_{w}\left(I_{Y}\right)$ is a monomial ideal of codimension $c$. Every minimal prime over $\mathrm{in}_{w}\left(I_{Y}\right)$ is generated by a subset of $c$ of the variables. We write $P_{\tau}=\left\langle x_{i}: i \in \tau\right\rangle$ for the monomial prime ideal indexed by the subset $\tau=\left\{\tau_{1}, \ldots, \tau_{c}\right\} \subset\{1,2, \ldots, n\}$.

Assume that the cone $w+\mathbb{R}_{>0}\left\{e_{\tau_{1}}, \ldots, e_{\tau_{c}}\right\}$ meets the tropicalization $\tau(Y)$. We may suppose that the generic weight vector $w \in \mathbb{R}^{n}$ satisfies the conditions that the image of $w$ in $\mathbb{T P}^{n-1}$ does not lie in $\tau(Y)$ and that the intersection of the cone $w+\mathbb{R}_{>0}\left\{e_{\tau_{1}}, \ldots, e_{\tau_{c}}\right\}$ with $\tau(Y)$ is finite and is contained in the union of the relative interiors of its maximal cones. Let $\sigma$ be a maximal cone of the tropical variety and let

$$
\{v\}=(L+w) \cap L^{\prime},
$$

where $L=\mathbb{R}\left\{e_{\tau_{1}}, \ldots, e_{\tau_{c}}\right\}$ and $L^{\prime}=\mathbb{R} \sigma$ are the corresponding linear spaces, which are defined over $\mathbb{Q}$. We associate with $v$ the lattice multiplicity of the intersection of $L$ and $L^{\prime}$, which is defined as the absolute value of the determinant of any $n \times n$ matrix whose columns consist of a $\mathbb{Z}$-basis of $\mathbb{Z}^{n} \cap L$ and a $\mathbb{Z}$-basis of $\mathbb{Z}^{n} \cap L^{\prime}$.

Here is the main result of this section.

Theorem 2.2. For $w \in \mathbb{R}^{n}$ a generic weight vector, a prime ideal $P_{\tau}$ is associated to the initial monomial ideal $\operatorname{in}_{w}\left(I_{Y}\right)$ if and only if the cone $w+\mathbb{R}_{>0}\left\{e_{\tau_{1}}, \ldots, e_{\tau_{c}}\right\}$ meets the tropicalization $\tau(Y)$. The number of intersections, each counted with its associated lattice multiplicity times the intrinsic multiplicity, is the multiplicity of the monomial ideal $\operatorname{in}_{w}\left(I_{Y}\right)$ along the prime $P_{\tau}$.

Proof. We work over the Puiseux series field $K=\mathbb{C}\left\{\left\{\epsilon^{\mathbb{R}}\right\}\right\}$, we write $K \mathbb{P}^{n-1}$ for the $(n-1)$-dimensional projective space over the field $K$, and we consider $Y$ as a subvariety of $K \mathbb{P}^{n-1}$. The translated variety $\epsilon^{-w} \cdot Y$ is defined by the prime ideal

$$
\epsilon^{w} \cdot I_{Y}=\left\{f\left(\epsilon^{w_{1}} x_{1}, \ldots, \epsilon^{w_{n}} x_{n}\right): f \in I_{Y}\right\} \quad \subset \quad K\left[x_{1}, \ldots, x_{n}\right] .
$$


Clearly, a point of the form $w+u$ lies in $\tau(Y)$ if and only if there is a point in $\epsilon^{-w} \cdot Y$ with order $u$.

Let $L$ be a general linear subspace of dimension $c$ in $K \mathbb{P}^{n-1}$ which is defined over $\mathbb{C}$. The intersection $\epsilon^{-w} \cdot Y \cap L$ is a finite set of reduced points in $K \mathbb{P}^{n-1}$. The number of these points is the degree $d$ of $Y$. There is a flat family over $K$ with generic fiber $\epsilon^{-w} \cdot Y$ and special fiber the scheme $V\left(\operatorname{in}_{w}\left(I_{Y}\right)\right)$. Since $L$ is generic, the intersection of $L$ with this family is still flat. Then, all points in the intersection $\epsilon^{-w} \cdot Y$ with $L$ are liftings of points in $V\left(\operatorname{in}_{w}\left(I_{Y}\right)\right) \cap L$ (and all points can be lifted). Moreover, the multiplicity of the $P_{\tau}$-primary component of the initial ideal $\operatorname{in}_{w}\left(I_{Y}\right)$ equals the number of points in $\epsilon^{-w} \cdot Y \cap L$ of the form

$$
\theta \cdot \epsilon^{u}+\ldots=\left(\theta_{1} \epsilon^{u_{1}}+\ldots: \theta_{2} \epsilon^{u_{2}}+\ldots: \cdots: \theta_{n} \epsilon^{u_{n}}+\ldots\right),
$$

where $\theta_{k} \in \mathbb{C}^{*}$ for all $k, u_{i}=0$ for $i \notin \tau$ and $u_{j}>0$ for $j \in \tau$, i.e., the number of points with values in the intersection of $\tau(Y)$ with the cone $w+\mathbb{R}_{>0}\left\{e_{\tau_{1}}, \ldots, e_{\tau_{c}}\right\}$.

By our genericity assumption, each such intersection point $v$ lies on some maximal cone $\sigma$ of $\tau(Y)$ and it is counted with its multiplicity, which is the product of the intrinsic multiplicity $m_{\sigma}$ times the lattice multiplicity of the transversal intersection of rational linear spaces in (2.2). This product can be understood by means of the flat family discussed in Remark 2.1 Namely, it follows from the $T$-invariance of the multiplication map $\Psi$ that the scheme-theoretic fiber of $\Psi$ over any point of $\mathcal{O}(\sigma)$ is isomorphic to $T^{\prime} \times Z_{\sigma}$, where $T^{\prime}$ is the stabilizer of a point in $\mathcal{O}(\sigma)$. Since $\mathbf{X}$ is normal, $T^{\prime}$ is a torus $\left(\mathbb{C}^{*}\right)^{r-1}$. By [24, 1.7], $\left(\mathbb{C}^{*}\right)^{r-1} \times Z_{\sigma}$ is the intersection of $T$ with the flat degeneration of $Y$ in $\mathbb{C P}^{n-1}$ given by the one parameter subgroup of $T$ specified by the rational vector $w$. Our construction above amounts to computing the intersection of $\left(\mathbb{C}^{*}\right)^{r-1} \times Z_{\sigma}$ with the corresponding degeneration of $L$. The lattice index is obtained from the factor $\left(\mathbb{C}^{*}\right)^{r-1}$, and $m_{\sigma}$ is obtained from the factor $Z_{\sigma}$. Their product is the desired intersection number.

The algebraic cycle of the variety $Y$ is represented by its Chow form $C h_{Y}$, which is a polynomial in the bracket variables $[\gamma]=\left[\gamma_{1} \cdots \gamma_{c}\right]$; see [13, §3.2.B]. Theorem 2.2 implies that the $w$-leading term of the Chow form $C h_{Y}$ equals $\prod[\gamma]^{u_{\gamma}}$, where $u_{\gamma}$ is the (correctly counted) number of points in $\tau(Y) \cap\left(w+\mathbb{R}_{>0}\left\{e_{\gamma_{1}}, \ldots, e_{\gamma_{c}}\right\}\right)$. We discuss this statement for the three families of examples considered earlier.

(1) If $Y$ is a hypersurface, then $c=1$ and the bracket variable $[\gamma]$ is simply the ordinary variable $x_{i}$ for $i=\gamma_{1}$. The $w$-leading monomial of the defining irreducible polynomial $f\left(x_{1}, \ldots, x_{n}\right)$ equals $x_{1}^{u_{1}} \cdots x_{n}^{u_{n}}$ where $u_{i}$ is the number of times the ray $w+\mathbb{R}_{>0} e_{i}$ intersects the tropical hypersurface $\tau(Y)$, counted with multiplicity.

(2) If $Y=X_{A}$ is a toric variety, then $\tau(Y)=\operatorname{rowspace}(A)$ and Theorem 2.2 implies the familiar result [13, Theorem 8.3.3] that the initial cycles of $X_{A}$ are the regular triangulations of $A$. Indeed, $w+\mathbb{R}_{>0}\left\{e_{\gamma_{1}}, \ldots, e_{\gamma_{n-d}}\right\}$ intersects the row space of $A$ if and only if the $(d-1)$-simplex $\bar{\gamma}=\left\{a_{i}: i \notin \gamma\right\}$ appears in the regular triangulation $\Pi_{w}$ of $A$ induced by $w$. For a precise definition of $\Pi_{w}$ see Section 5. The intersection multiplicity is the lattice volume of $\bar{\gamma}$. Hence $\operatorname{in}_{w}\left(C h_{X_{A}}\right)=\prod_{\bar{\gamma} \in \Pi_{w}}[\gamma]^{\operatorname{vol}(\bar{\gamma})}$.

(3) If $Y$ is a linear space, then its ideal $I_{Y}$ is generated by $c$ linearly independent linear forms in $\mathbb{C}\left[x_{1}, \ldots, x_{n}\right]$. The tropical variety $\tau(Y)$ is the Bergman fan, to be discussed in Section 3, and the Gröbner fan of $I_{Y}$ is the normal fan of the associated matroid polytope [10]. For fixed generic $w$, there is a unique $c$-element subset $\gamma$ 
of $[n]$ such that $w+\mathbb{R}_{>0}\left\{e_{\gamma_{1}}, \ldots, e_{\gamma_{c}}\right\}$ intersects $\tau(Y)$. The intersection multiplicity is one, and the corresponding initial ideal equals $\operatorname{in}_{w}\left(I_{Y}\right)=\left\langle x_{\gamma_{1}}, \ldots, x_{\gamma_{c}}\right\rangle$.

\section{TROPICALIZING MAPS DEFINED BY MONOMIALS IN LINEAR FORMS}

In this section we examine a class of rational varieties $Y$ whose tropicalization $\tau(Y)$ can be computed combinatorially, without knowing the ideal $I_{Y}$. We consider a rational map $f: \mathbb{C}^{m} \rightarrow \mathbb{C}^{s}$ that factors as a linear map $\mathbb{C}^{m} \rightarrow \mathbb{C}^{r}$ followed by a Laurent monomial map $\mathbb{C}^{r} \rightarrow \mathbb{C}^{s}$. The linear map is specified by a complex $r \times m$-matrix $U=\left(u_{i j}\right)$, and the Laurent monomial map is specified by an integer $s \times r$-matrix $V=\left(v_{i j}\right)$. Thus the $i$ th coordinate of the rational map $f: \mathbb{C}^{m} \rightarrow \mathbb{C}^{s}$ equals the following monomial in linear forms:

$$
f_{i}\left(x_{1}, \ldots, x_{m}\right)=\prod_{k=1}^{r}\left(u_{k 1} x_{1}+\cdots+u_{k m} x_{m}\right)^{v_{i k}}, \quad i=1, \ldots, s .
$$

Let $Y_{U V}$ denote the Zariski closure of the image of $f$. Observe that if all row sums of $V$ are equal, then $f$ induces a rational map $\mathbb{C P}^{m-1} \rightarrow \rightarrow \mathbb{C P}^{s-1}$, and the closure of its image is an irreducible projective variety, which we also denote by $Y_{U V}$.

Our goal is to compute the tropicalization $\tau\left(Y_{U V}\right)$ of the variety $Y_{U V}$ in terms of the matrices $U$ and $V$. In particular, we will avoid any reference to the equations of $Y_{U V}$. The general framework of this section will be crucial for our proofs of the results on $A$-discriminants and their tropicalization stated in the Introduction.

We list a number of special cases of varieties which have the form $Y_{U V}$.

(1) If $r=s$ and $V=I_{r}$, then $f$ is the linear map $x \mapsto U x$ and $Y_{U V}$ is the image of $U$. We denote this linear subspace of $\mathbb{C}^{r}$ by $\operatorname{im}(U)$. Its tropicalization $\tau(\operatorname{im}(U))$ is the Bergman fan of the matrix $U$, to be discussed in detail below.

(2) If $m=r$ and $U=I_{m}$, then $f$ is the monomial map specified by the matrix $V$. Hence $Y_{U V}$ coincides with the toric variety $X_{V^{t}}$ which is associated with the transpose $V^{t}$ of the matrix $V$. Its tropicalization is the column space of $V$.

(3) Let $m=2$, suppose the rows of $U$ are linearly independent, and suppose the matrix $V$ has constant row sum. Then $Y_{U V}=$ image $\left(\mathbb{C P}^{1} \rightarrow \mathbb{C P}^{s-1}\right)$ is a rational curve. Every rational projective curve arises from this construction, since every binary form is a monomial in linear forms.

Our next theorem implies that $\tau\left(Y_{U V}\right)$ consists of the rays in $\mathbb{T} \mathbb{P}^{s-1}$ spanned by the rows of $V$. Theorem 3.1 can also be derived from [24, Proposition 3.1], but we prefer to give a proof that is entirely self-contained.

Theorem 3.1. The tropical variety $\tau\left(Y_{U V}\right)$ equals the image of the Bergman fan $\tau(\operatorname{im}(U))$ under the linear map $\mathbb{R}^{r} \rightarrow \mathbb{R}^{s}$ specified by the matrix $V$.

Proof. In what follows, we consider all algebraic varieties over the field $K=\mathbb{C}\left\{\left\{\epsilon^{\mathbb{R}}\right\}\right\}$ and we use the characterization of the tropical variety $\tau\left(Y_{U V}\right)$ in terms of $K^{*}$-valued points of the ideal of $Y_{U V}$. Extending scalars, we consider the map $f: K^{m} \rightarrow K^{s}$. Let $z=f(x)$ be any point in the image of that map. For $k \in\{1, \ldots, r\}$ we set $y_{k}=u_{k 1} x_{1}+\cdots+u_{k m} x_{m}$ and $\alpha_{k}=\operatorname{order}\left(y_{k}\right)$. The vector $y=\left(y_{1}, \ldots, y_{r}\right)$ lies in the linear space $\operatorname{im}(U)$, and hence the vector $\alpha=\left(\alpha_{1}, \ldots, \alpha_{r}\right)$ lies in $\tau(\operatorname{im}(U))$.

The order of the vector $z=f(x) \in K^{s}$ is the vector $V \cdot \alpha \in \mathbb{R}^{s}$, since the order of its $i$ th coordinate $z_{i}$ equals $\sum_{k=1}^{r} v_{j k} \cdot \alpha_{k}$. Hence $z=f(x)$ lies in $V \cdot \tau(\operatorname{im}(U))$, the image of the Bergman fan $\tau(\operatorname{im}(U))$ under the linear map $V$. 
The image of $f$ is Zariski dense in $Y_{U V}$, i.e., there exists a proper subvariety $Y$ of $Y_{U V}$ such that $Y_{U V} \backslash Y$ contains the image of $f$. By the Bieri-Groves Theorem [2], $\tau(Y)$ is a polyhedral fan of lower dimension inside the pure-dimensional fan $\tau\left(Y_{U V}\right)$. From this it follows that $\tau\left(Y_{U V}\right)$ is the closure of $\tau\left(Y_{U V} \backslash Y\right)$ in $\mathbb{R}^{s}$. In the previous paragraph we showed that $\tau\left(Y_{U V} \backslash Y\right)$ is a subset of $V \cdot \tau(\operatorname{im}(U))$. Since the latter is closed, we conclude that $\tau\left(Y_{U V}\right) \subseteq V \cdot \tau(\operatorname{im}(U))$.

It remains to show the converse inclusion $V \cdot \tau(\operatorname{im}(U)) \subseteq \tau\left(Y_{U V}\right)$. Take any point $\beta \in V \cdot \tau(\operatorname{im}(U))$, and choose $\alpha \in \tau(\operatorname{im}(U))$ such that $V \cdot \alpha=\beta$. There exists a $K^{*}$-valued point $y$ in the linear space $\operatorname{im}(U)$ such that $\operatorname{order}(y)=\alpha$. Then the point $y^{V}=\left(\prod_{k=1}^{r} y_{k}^{v_{1 k}}, \ldots, \prod_{k=1}^{r} y_{k}^{v_{s k}}\right)$ lies in $\left(K^{*}\right)^{s} \cap Y_{U V}$, and its order clearly equals $\beta$. Hence $\beta \in \tau\left(Y_{U V}\right)$ as desired.

Remark 3.2. The intrinsic multiplicity $m_{\sigma}$ of any maximal cone $\sigma$ in a sufficiently fine fan structure on the tropical variety $\tau\left(Y_{U V}\right)$ is a lattice index which can be read off from the matrix $V$. Namely, suppose $\sigma$ lies the image of a maximal cone $\sigma^{\prime}$ of the Bergman fan $\tau(\operatorname{im}(U))$. Then $m_{\sigma}$ is the index of the subgroup $V\left(\mathbb{R} \sigma^{\prime} \cap \mathbb{Z}^{r}\right)$ of $\mathbb{R} \sigma \cap \mathbb{Z}^{s}$. This follows from Remark 2.1 using standard arguments of toric geometry.

Theorem 3.1 and Remark 3.2 furnish a combinatorial construction for the tropicalization of any variety which is parameterized by monomials in linear forms. Using the results of Section 2. Theorem 3.1 can now be applied to compute geometric invariants of such a variety, for instance, its dimension, its degree and its initial cycles. To make this computation effective, we need an explicit description of the Bergman fan $\tau(\operatorname{im}(U))$. Luckily, the relevant combinatorics is well understood, thanks to [1, 10, and in the remainder of this section we summarize what is known.

Let $M$ denote the matroid associated with the rows of the $r \times m$-matrix $U$. Thus $M$ is a matroid of rank at most $m$ on the ground set $[r]=\{1,2, \ldots, r\}$. The bases of $M$ are the maximal subsets of $[r]$ which index linearly independent rows of $U$. Fix a vector $w \in \mathbb{R}^{r}$. Then the $w$-weight of a basis $\beta$ of $M$ is $\sum_{i \in \beta} w_{i}$. Consider the set of all bases of $M$ that have maximal $w$-weight. This collection is the set of bases of a new matroid which we denote by $M_{w}$. Note that each $M_{w}$ has the same rank and the same ground set as $M=M_{0}$. An element $i$ of $[r]$ is a loop of $M_{w}$ if it does not lie in any basis of $M$ of maximal $w$-weight.

We can now describe $\tau(\operatorname{im}(U))$ in terms of the matroid $M$ :

$$
\tau(\operatorname{im}(U))=\left\{w \in \mathbb{R}^{r}: M_{w} \text { has no loop }\right\}
$$

This representation endows our tropical linear space with the structure of a polyhedral fan. Namely, if $w \in \tau(\operatorname{im}(U))$, then the set of all $w^{\prime} \in \mathbb{R}^{r}$ such that $M_{w^{\prime}}=M_{w}$ is a relatively open convex polyhedral cone in $\mathbb{R}^{r}$. The collection of these cones is denoted $\mathcal{B}(M)$ and is called the Bergman fan of the matroid $M$. Depending on the context, we may also write $\mathcal{B}(U)$ and call it the Bergman fan of the matrix $U$.

We now recall the connection between Bergman fans and nested set complexes. The latter encode the structure of wonderful compactifications of hyperplane arrangement complements in the work of De Concini and Procesi [5], and they were later studied from a combinatorial point of view in [8, 9, 11.

A subset $X \subseteq[r]$ is a flat of our matroid $M$ if there exists a vector $u \in \operatorname{im}(U)$ such that $X=\left\{i \in[r]: u_{i}=0\right\}$. The set of all flats, ordered by inclusion, is the geometric lattice $\mathcal{L}=\mathcal{L}_{M}$. A flat $X$ in $\mathcal{L}$ is called irreducible if the lower interval $\{Y \in \mathcal{L}: Y \leq X\}$ does not decompose as a direct product of posets. Denote by $\mathcal{I}$ the set of irreducible elements in $\mathcal{L}$. In other contexts, the irreducible elements of 
a lattice of flats of a matroid were named connected elements or dense edges. The matroid $M$ is connected if the top rank flat $\hat{1}=[r]$ is irreducible, and we assume that this is the case. Otherwise, we artificially add $\hat{1}$ to $\mathcal{I}$. We call a subset $S \subseteq \mathcal{I}$ nested if for any set of pairwise incomparable elements $X_{1}, \ldots, X_{t}$ in $S$, with $t \geq 2$, the join $X_{1} \vee \ldots \vee X_{t}$ is not contained in $\mathcal{I}$. The nested subsets in $\mathcal{I}$ form a simplicial complex, the nested set complex $\mathcal{N}(\mathcal{L})$. See [8, Section 2.3] for further information.

Feichtner and Yuzvinsky [11, equation (13)] introduced the following natural geometric realization of the nested set complex $\mathcal{N}(\mathcal{L})$. Namely, the collection of cones

$$
\mathbb{R}_{\geq 0}\left\{e_{X}: X \in S\right\} \quad \text { for } S \in \mathcal{N}(\mathcal{L})
$$

forms a unimodular fan whose face poset is the face poset of the nested set complex of $\mathcal{L}$. Here $e_{X}=\sum_{i \in X} e_{i}$ denotes the incidence vector of a flat $X \in \mathcal{I}$. We consider this fan in the tropical projective space $\mathbb{T} \mathbb{P}^{n-1}$, and we also denote it by $\mathcal{N}(\mathcal{L})$.

It was shown in [10, Theorem 4.1] that the nested set fan $\mathcal{N}\left(\mathcal{L}_{M}\right)$ is a simplicial subdivision of the Bergman fan $\mathcal{B}(M)$, and hence of our tropical linear space $\tau(\operatorname{im}(U))$. The Bergman fan need not be simplicial, so the nested set fan can be finer than the Bergman fan. However, in many important cases the two fans coincide [10, §5].

What we defined above is the coarsest in a hierarchy of nested set complexes associated with the geometric lattice $\mathcal{L}$. Namely, for certain choices of subsets $\mathcal{G}$ in $\mathcal{L}$, the same construction gives a nested set complex $\mathcal{N}(\mathcal{L}, \mathcal{G})$ which is also realized as a unimodular simplicial fan. Such $\mathcal{G}$ are called building sets; there is one nested set fan for each building set $\mathcal{G}$ in $\mathcal{L}$ (see [8. Section 2.2, 2.3]). If two building sets are contained in another, $\mathcal{G}_{1} \subseteq \mathcal{G}_{2}$, then $\mathcal{N}\left(\mathcal{L}, \mathcal{G}_{2}\right)$ is obtained from $\mathcal{N}\left(\mathcal{L}, \mathcal{G}_{1}\right)$ by a sequence of stellar subdivisions [9, Theorem 4.2]. The smallest building set is $\mathcal{G}=\mathcal{I}$, the case discussed above, and the largest building set is the set of all flats, $\mathcal{G}=\mathcal{L}$. In the letter case, the corresponding nested set complex $\mathcal{N}(\mathcal{L}, \mathcal{L})$ is the order complex or flag complex $\mathcal{F}(\mathcal{L})$ of the lattice, i.e., the simplicial complex on $\mathcal{L} \backslash\{\hat{0}\}$ whose simplices are the totally ordered subsets. Again, there is a realization of $\mathcal{F}(\mathcal{L})$ as a unimodular fan with rays generated by the incidence vectors of flats in $\mathcal{L}$. We call this fan the flag fan of $\mathcal{L}$ and denote it by $\mathcal{F}(\mathcal{L})$ for ease of notation.

Summarizing the situation, we have the following sequence of subdivisions each of which can be used to compute the tropicalization of a linear space.

Theorem 3.3. Given a matrix $U$ and the matroid $M$ of rows in $U$, the tropical linear space $\tau(\operatorname{im}(U))$ has three natural fan structures: the Bergman fan $\mathcal{B}(M)$ is refined by the nested set fan $\mathcal{N}\left(\mathcal{L}_{M}\right)$, which is refined by the flag fan $\mathcal{F}\left(\mathcal{L}_{M}\right)$.

We present an example which illustrates the concepts developed in this section.

Example 3.4. Let $m=4, r=5, s=4$, and consider the map $f=\left(f_{1}, f_{2}, f_{3}, f_{4}\right)$ from $\mathbb{C}^{4}$ to $\mathbb{C}^{4}$ whose coordinates are the following monomials in linear forms:

$$
\begin{aligned}
& f_{1}=\left(x_{1}-x_{2}\right)^{3}\left(x_{1}-x_{3}\right)^{3}, \\
& f_{2}=\left(x_{1}-x_{2}\right)^{2}\left(x_{2}-x_{3}\right)^{2}\left(x_{2}-x_{4}\right)^{2}, \\
& f_{3}=\left(x_{1}-x_{3}\right)^{2}\left(x_{2}-x_{3}\right)^{2}\left(x_{3}-x_{4}\right)^{2}, \\
& f_{4}=\left(x_{2}-x_{4}\right)^{3}\left(x_{3}-x_{4}\right)^{3} .
\end{aligned}
$$


So $f$ is as in (3.1) with

$$
U=\left(\begin{array}{rrrr}
1 & -1 & 0 & 0 \\
1 & 0 & -1 & 0 \\
0 & 1 & -1 & 0 \\
0 & 1 & 0 & -1 \\
0 & 0 & 1 & -1
\end{array}\right) \text { and } V=\left(\begin{array}{lllll}
3 & 3 & 0 & 0 & 0 \\
2 & 0 & 2 & 2 & 0 \\
0 & 2 & 2 & 0 & 2 \\
0 & 0 & 0 & 3 & 3
\end{array}\right)
$$

The projectivization of the variety $Y_{U V}=\operatorname{cl}(\operatorname{image}(f))$ is a surface in $\mathbb{C P}^{3}$, since $U$ has rank 3 . We construct the irreducible homogeneous polynomial $P\left(z_{1}, z_{2}, z_{3}, z_{4}\right)$ which defines this surface. The matroid of $U$ is the graphic matroid of $K_{4}$ with one edge removed. From [10, Example 3.4] we know that the Bergman complex $\mathcal{B}(U)$ is the complete bipartite graph $K_{3,3}$, embedded as a 2-dimensional fan in $\mathbb{T P}^{4}$.

The tropical surface $\tau\left(Y_{U V}\right)$ is the image of $\mathcal{B}(U)$ under $V$. This image is a 2-dimensional fan in $\mathbb{P P}^{3}$. It has seven rays: six of them are images of the rays of $\mathcal{B}(U)$, and the last one is the intersection of the images of two 2-dimensional faces that occurs due to the non-planarity of $K_{3,3}$. Hence the Newton polytope of the polynomial $P$ is 3 -dimensional with 6 vertices, 11 edges, and 7 facets; see Figure 1

The six extreme monomials of $P$ can be computed (even by hand) using Theorem 2.2. namely, by intersecting the rays $w+\mathbb{R}_{>0} e_{i}$ with $\tau\left(Y_{U V}\right)$ in $\mathbb{T P}^{3}$. This computation reveals in particular that the degree of the polynomial $P$ is 28 . Using linear algebra, it is now easy to determine all 171 monomials in the expansion of $P$.

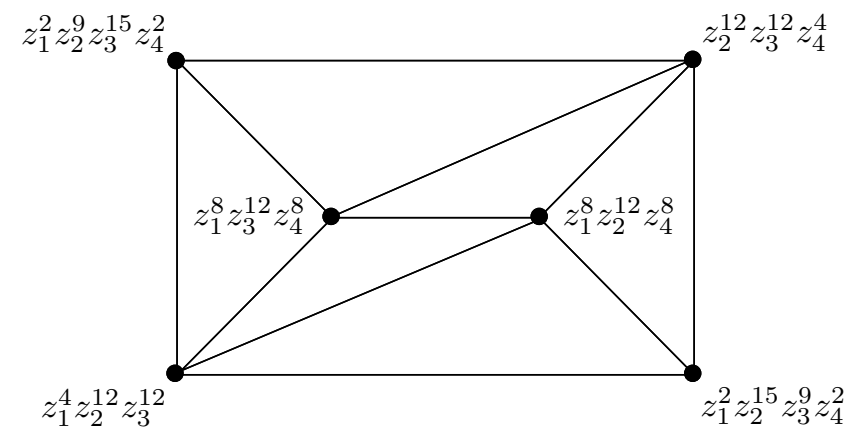

Figure 1. The Newton polytope of the polynomial $P$ in Example 3.4

\section{BACK to $A$-DisCRIminants}

In this section we return to the setting of the Introduction, and we prove Theorem 1.1 and Theorem 1.2. Recall that $A$ is an integer $d \times n$-matrix such that $(1, \ldots, 1)$ is in the row span of $A$, i.e., the column vectors $a_{1}, a_{2}, \ldots, a_{n}$ lie in an affine hyperplane in $\mathbb{R}^{d}$. We also assumed that the vectors $a_{1}, \ldots, a_{n}$ span $\mathbb{Z}^{d}$. We identify the matrix $A$ with the point configuration $\left\{a_{1}, a_{2}, \ldots, a_{n}\right\}$. The convex hull of the configuration $A$ is a $(d-1)$-dimensional polytope with $\leq n$ vertices.

The projective toric variety $X_{A}$ is defined as the closure of the image of the monomial map $\psi_{A}:\left(\mathbb{C}^{*}\right)^{d} \rightarrow \mathbb{C P}^{n-1}, t \mapsto\left(t^{a_{1}}: t^{a_{2}}: \cdots: t^{a_{n}}\right)$. Equivalently, $X_{A}$ is the set of all points $x \in \mathbb{C P}^{n-1}$ such that $x^{u}=x^{v}$ for all $u, v \in \mathbb{N}^{n}$ with $A u=A v$. 
Let $\left(\mathbb{C P}^{n-1}\right)^{*}$ denote the projective space dual to $\mathbb{C P}^{n-1}$. The point $\xi=\left(\xi_{1}: \cdots\right.$ : $\left.\xi_{n}\right)$ in $\left(\mathbb{C P}^{n-1}\right)^{*}$ corresponds to the hyperplane $H_{\xi}=\left\{x \in \mathbb{C P}^{n-1}: \sum_{i=1}^{n} x_{i} \xi_{i}=\right.$ $0\}$. The dual variety $X_{A}^{*}$ is defined as the closure in $\left(\mathbb{C P}^{n-1}\right)^{*}$ of the set of points $\xi$ such that the hyperplane $H_{\xi}$ intersects the toric variety $X_{A}$ at a regular point $p$ and contains the tangent space $T_{X_{A}}(p)$ of $X_{A}$ at $p$.

Kapranov [16] showed that reduced discriminantal varieties are parametrized by monomials in linear forms. This parametrization, called the Horn uniformization, will allow us to determine the tropical discriminant $\tau\left(X_{A}^{*}\right)$ via the results of Section 3 . We denote by $\mathbb{C P}(\operatorname{ker}(A))$ the projectivization of the kernel of the linear map given by $A$, an $(n-d-1)$-dimensional projective subspace of $\mathbb{C P}^{n-1}$, and we denote by $T^{d-1}=\left(\mathbb{C}^{*}\right)^{d} / \mathbb{C}^{*}$ the dense torus of $X_{A}$. The following result is a variant of [16, Theorem 2.1]; see also [13, §9.3.C].

Proposition 4.1. The dual variety $X_{A}^{*}$ of the toric variety $X_{A}$ is the closure of the image of the map $\varphi_{A}: \mathbb{C P}(\operatorname{ker}(A)) \times T^{d-1} \rightarrow\left(\mathbb{C P}^{n-1}\right)^{*}$ which is given by

$$
\varphi_{A}(u, t)=\left(u_{1} t^{a_{1}}: u_{2} t^{a_{2}}: \cdots: u_{n} t^{a_{n}}\right) .
$$

Proof. Consider the unit point $1=(1: 1: \ldots: 1)$ on the toric variety $X_{A}$. The hyperplane $H_{\xi}$ contains both the point 1 and the tangent space $T_{X_{A}}(\mathbf{1})$ at this point if and only if $\xi$ lies in the kernel of $A$. This follows by evaluating the derivative of the parametrization $\psi_{A}$ of $X_{A}$ at $\left(t_{1}, t_{2}, \ldots, t_{d}\right)=(1,1, \ldots, 1)$. If $p=\psi_{A}(t)$ is any point in the dense torus of $X_{A}$, then the tangent space at that point is obtained by translating the tangent space at $\mathbf{1}$ as follows:

$$
T_{X_{A}}(p)=p \cdot T_{X_{A}}(\mathbf{1}) .
$$

The hyperplane $H_{\xi}$ contains $p$ if and only if $p^{-1} \cdot H_{\xi}=H_{\xi \cdot p}$ contains $\mathbf{1}$, and $H_{\xi}$ contains $T_{X_{A}}(p)$ if and only if $H_{\xi \cdot p}$ contains $T_{X_{A}}(\mathbf{1})$. These two conditions hold, for some $p$ in the dense torus of $X_{A}$, if and only if $\xi \in \operatorname{image}\left(\varphi_{A}\right)$.

Proposition 4.1 shows that the dual variety $X_{A}^{*}$ of the toric variety $X_{A}$ is parametrized by monomials in linear forms. In the notation of Section 3 we set $m=n, r=n+d, s=n$, and the two matrices are

$$
U=\left(\begin{array}{cc}
B & 0 \\
0 & I_{d}
\end{array}\right) \text { and } V=\left(\begin{array}{ll}
I_{n} & A^{t}
\end{array}\right),
$$

where $B$ is an $n \times(n-d)$-matrix whose columns span the kernel of $A$ over the integers. Thus the rows of $B$ are Gale dual to the configuration $A$.

Lemma 4.2. The variety $Y_{U V} \subset \mathbb{C}^{n}$ defined by (4.2) as in Section 3 is equal to the cone over the dual variety $X_{A}^{*} \subset\left(\mathbb{C P}^{n-1}\right)^{*}$ of the toric variety $X_{A} \subset \mathbb{C P}^{n-1}$.

Proof. Let $f$ be the rational map defined by (4.2) as in Section 3 and set $x=$ $\left(x_{1}, \ldots, x_{n-d}\right)$ and $t=\left(t_{1}, \ldots, t_{d}\right)=\left(x_{n-d+1}, \ldots, x_{n}\right)$. Then (3.1) equals

$$
f_{i}(x, t)=\left(b_{i, 1} x_{1}+\cdots+b_{i, n-d} x_{n-d}\right) \cdot t^{a_{i}},
$$

which equals the $i$ th coordinate of $\varphi_{A}$ if we write $\operatorname{ker}(A)$ as the image of $B$.

We are now ready to prove Theorem 1.1 .

Proof of Theorem 1.1. Let us first note that the co-Bergman fan $\mathcal{B}^{*}(A)$ of the rank $d$ configuration given by the columns of $A$ equals the Bergman fan of the rank $n-d$ configuration given by the rows of $B$. Thus $\mathcal{B}^{*}(A)$, as it appears in (1.1), is the Bergman fan of the matroid dual to the matroid given by the columns of $A$. 
The support of the co-Bergman fan $\mathcal{B}^{*}(A)$ is the tropicalization of the linear space $\operatorname{ker}(A)=\operatorname{im}(B)$. Now, if $U$ is taken as in (4.2), then we have the following decomposition in $\mathbb{R}^{r}=\mathbb{R}^{n} \oplus \mathbb{R}^{d}$ :

$$
\tau(\operatorname{im}(U))=\tau(\operatorname{im}(B)) \oplus \tau\left(\operatorname{im}\left(I_{d}\right)\right)=\mathcal{B}^{*}(A) \oplus \mathbb{R}^{d} .
$$

The image of this fan under the linear map $V=\left(\begin{array}{ll}I_{n} & A^{t}\end{array}\right)$ is the (Minkowski) sum of $\mathcal{B}^{*}(A)$ and the image of $A^{t}$. Of course, the latter is the row space of $A$. Hence our assertion follows from Lemma 4.2 and Theorem 3.1

Similarly, the tropicalization of the reduced version of the dual variety can be derived from Theorem 3.1. Let $B$ be again an $n \times(n-d)$-matrix whose columns span the kernel of $A$ over the integers. The reduced dual variety $Y_{B}^{*}:=Y_{B B^{t}}$ is the closure of the image of the rational morphism $\widetilde{\varphi}_{B}: \mathbb{C P}^{n-d-1} \rightarrow \mathbb{C}^{n-d}$, whose $i$ th coordinate equals

$$
\widetilde{\varphi}_{B}\left(s_{1}: \ldots: s_{n-d}\right)_{i}=\prod_{k=1}^{n}\left(b_{k, 1} s_{1}+\ldots+b_{k, n-d} s_{n-d}\right)^{b_{k, i}}, \quad i=1, \ldots, n-d .
$$

Corollary 4.3. Given a Gale dual $B$ of $A$, the tropicalization of the reduced dual variety $Y_{B}^{*}$ is the image of the co-Bergman fan $\mathcal{B}^{*}(A)$ under the linear map $B^{t}$.

We often do not distinguish between the reduced and unreduced version of dual varieties and their tropicalizations, and we denote both by $X_{A}^{*}$ and $\tau\left(X_{A}^{*}\right)$, respectively.

We illustrate Theorem 1.1 for the case when $X_{A}$ is the Veronese surface, regarded as the projectivization of the variety of all symmetric $3 \times 3$ matrices of rank $\leq 1$.

Example 4.4. We take $d=3, n=6$, and we fix the matrix

$$
A=\left(\begin{array}{llllll}
1 & 1 & 1 & 1 & 1 & 1 \\
0 & 1 & 2 & 0 & 1 & 0 \\
0 & 0 & 0 & 1 & 1 & 2
\end{array}\right)
$$

Note that the more usual matrix

$$
A^{\prime}=\left(\begin{array}{llllll}
2 & 1 & 0 & 1 & 0 & 0 \\
0 & 1 & 2 & 0 & 1 & 0 \\
0 & 0 & 0 & 1 & 1 & 2
\end{array}\right)=\left(\begin{array}{rrr}
2 & -1 & -1 \\
0 & 1 & 0 \\
0 & 0 & 1
\end{array}\right) \cdot A
$$

defines the same Veronese embedding of $\mathbb{C P}^{2}$ into $\mathbb{C P}^{5}$, but the columns of $A^{\prime}$ do not span $\mathbb{Z}^{3}$ and the monomial parametrization $\psi_{A^{\prime}}$ is two-to-one.

Points $x$ in $\mathbb{C P}^{5}$ are identified with symmetric $3 \times 3$-matrices

$$
X=\left(\begin{array}{ccc}
2 x_{1} & x_{2} & x_{4} \\
x_{2} & 2 x_{3} & x_{5} \\
x_{4} & x_{5} & 2 x_{6}
\end{array}\right)
$$

A point $u$ is in $\mathbb{C P}(\operatorname{ker}(A))$ if and only if the corresponding matrix $U$ has zero row and column sums. If this holds and if $t$ is any point in $\left(\mathbb{C}^{*}\right)^{3}$, then the symmetric $3 \times 3$-matrix $X$ corresponding to $x=\varphi_{A}(u, t)$ is singular because it satisfies

$$
\left(\begin{array}{lll}
1 & 1 / t_{2} & 1 / t_{3}
\end{array}\right) \cdot X=\left(\begin{array}{lll}
0 & 0 & 0
\end{array}\right) .
$$

Hence $\varphi_{A}$ parametrizes rationally the hypersurface of singular symmetric matrices $X$, and the $A$-discriminant equals the classical discriminant $\Delta_{A}(x)=\frac{1}{2} \operatorname{det}(X)$. 

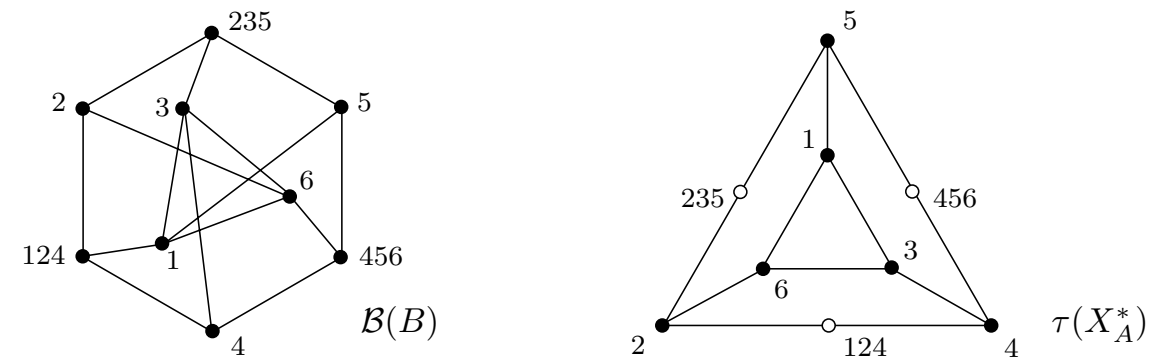

Figure 2. Bergman complex and tropical discriminant in Example 4.4

The tropicalization of $X_{A}^{*}$ is obtained as follows. We choose a Gale dual $B$ of $A$,

$$
B^{t}=\left(\begin{array}{rrrrrr}
1 & -2 & 1 & 0 & 0 & 0 \\
1 & -1 & 0 & -1 & 1 & 0 \\
1 & 0 & 0 & -2 & 0 & 1
\end{array}\right) \text {. }
$$

Note that the matroid given by the columns of $A$ is self-dual. The Bergman fan $\mathcal{B}(B)=\mathcal{B}^{*}(A)$ is a 2 -dimensional fan in $\mathbb{R}^{6} / \mathbb{R}(1,1,1,1,1,1)$, or, equivalently, a graph on the 4-sphere. We depict this graph in Figure 2 on the left. It has nine vertices, corresponding to the six singletons $1,2, \ldots, 6$ and the three circuits $124,235,456$.

Its image under $B^{t}$ is the tropical discriminant $\tau\left(X_{A}^{*}\right)$, a 2-dimensional fan in 3-dimensional real space or, equivalently, a graph on the 2 -sphere. We depict this graph in Figure 2 on the right. The rays 124, 235, and 456 of $\mathcal{B}(B)$ map into the relative interiors of the 2-dimensional cones 24,25 , and 45 of $\tau\left(X_{A}^{*}\right)$, respectively. More precisely, the ray 124 of $\mathcal{B}(B)$ is mapped to the negative of the image of the ray 1 , which in turn is the dividing ray in the 2-dimensional cone spanned by the images of the rays 2 and 4 . The rays 235 and 456 are mapped similarly.

Since $A$ is non-defective, the tropical discriminant $\tau\left(X_{A}^{*}\right)$ is the union of codimension 1 cones in the normal fan of the Newton polytope of $\Delta_{A}$. As $\tau\left(X_{A}^{*}\right)$ is the 1-skeleton of a triangular prism, we conclude that the Newton polytope of $\Delta_{A}$ is a bipyramid. Its five vertices correspond to the five terms in the determinant of $X$.

Returning to the general case, we note that the dimension of the image of $\varphi_{A}$ is at most $\operatorname{dim}\left(\mathbb{C P}(\operatorname{ker}(A)) \times T^{d-1}\right)=n-2$, so the dual variety $X_{A}^{*}$ is a proper subvariety of $\mathbb{C P}^{n-1}$. If the dimension of $X_{A}^{*}$ is less than $n-2$, that is, if $X_{A}^{*}$ is not a hypersurface, we say that the toric variety $X_{A}$ and its point configuration $A$ are defective. In the non-defective case, there is a unique (up to sign) irreducible polynomial $\Delta_{A}$ with integer coefficients which vanishes on $X_{A}^{*}$. The polynomial $\Delta_{A}$ is the $A$-discriminant as defined in [13, $99.1 . \mathrm{A}]$. In what follows, the dual variety $X_{A}^{*}$ itself will be referred to as the $A$-discriminant, even if $A$ is defective.

By the Bieri-Groves Theorem [2, 23], the dimension of the $A$-discriminant $X_{A}^{*}$ coincides with the dimension of the tropical $A$-discriminant $\tau\left(X_{A}^{*}\right)$. Theorem 1.1 furnishes a purely combinatorial formula for that dimension.

Corollary 4.5. The dimension of the A-discriminant $X_{A}^{*}$ in $\mathbb{C P}^{n-1}$ is one less than the largest rank of any matrix $\left(A^{t}, \sigma_{1}, \ldots, \sigma_{n-d-1}\right)$ where $\sigma$ runs over $\mathcal{C}(A)$.

Here $\mathcal{C}(A)$ is the subset of $\{0,1\}^{n}$ defined in the Introduction. That definition is now best understood using the matroid-theoretic concepts which we reviewed in the 
second half of Section 3, where we take $U$ to be the $n \times(n-d)$-matrix $B$ as in (4.2) and, hence, $M$ to be the rank $n-d$ matroid associated with the rows of $B$. In fact, $M$ is the matroid dual to the matroid given by the columns of the $d \times n$-matrix $A$, and $\mathcal{L}(A)$ coincides with the lattice of flats $\mathcal{L}_{M}$. The set $\mathcal{C}(A)$ corresponds to the facets of the flag complex $\mathcal{F}\left(\mathcal{L}_{M}\right)$. In light of Theorem 3.3, one could reformulate Corollary 4.5 with $\sigma$ ranging over the facets of the nested set complex $\mathcal{N}\left(\mathcal{L}_{M}\right)$ or the Bergman fan $\mathcal{B}(M)=\mathcal{B}^{*}(A)$.

We are now prepared to state and prove the general version of Theorem 1.2 , Let $\mathcal{C}^{c}$ denote the set of all proper chains of length $n-d-c-1$ in $\mathcal{L}(A)=\mathcal{L}_{M}$, where we identify flats of the matroid in $\mathcal{L}(A)$ with their incidence vectors, and hence the chains in $\mathcal{C}^{c}$ with $(n-d-c)$-element subsets of $\{0,1\}^{n}$. Equivalently, $\mathcal{C}^{c}$ is the set of $(n-d-c)$-element subsets of the elements of $\mathcal{C}=\mathcal{C}(A)$. We write $\operatorname{in}_{w}\left(X_{A}^{*}\right)=\operatorname{in}_{w}\left(I_{X_{A}^{*}}\right)$ for the initial ideal, with respect to some $w \in \mathbb{R}^{n}$, of the homogeneous prime ideal $I_{X_{A}^{*}}$ of the $A$-discriminant $X_{A}^{*}$.

Theorem 4.6. Suppose that the A-discriminant $X_{A}^{*}$ has codimension $c$ and let $\tau=\left\{\tau_{1}, \ldots, \tau_{c}\right\} \subset\{1, \ldots, n\}$. If $w$ is a generic vector in $\mathbb{R}^{n}$, then the multiplicity of the initial monomial ideal $\operatorname{in}_{w}\left(X_{A}^{*}\right)$ along the prime $P_{\tau}=\left\langle x_{i}: i \in \tau\right\rangle$ equals

$$
\sum_{\sigma \in \mathcal{C}_{i, w}^{c}}\left|\operatorname{det}\left(A^{t}, \sigma_{1}, \ldots, \sigma_{n-d-c}, e_{\tau_{1}}, \ldots, e_{\tau_{c}}\right)\right|,
$$

where $\mathcal{C}_{i, w}^{c}$ is the subset of $\mathcal{C}^{c}$ consisting of all chains $\sigma$ such that

$$
\operatorname{rowspace}(A) \cap \mathbb{R}_{>0}\left\{\sigma_{1}, \ldots, \sigma_{n-d-c},-e_{\tau_{1}}, \ldots,-e_{\tau_{c}},-w\right\} \neq \emptyset .
$$

Theorem 1.2 is the special case of Theorem 4.6 when $A$ is non-defective, i.e., $c=1$ and $I_{X_{A}^{*}}$ is the principal ideal generated by $\Delta_{A}$. In that case, the initial monomial ideal $\operatorname{in}_{w}\left(X_{A}^{*}\right)$ is generated by the initial monomial $\operatorname{in}_{w}\left(\Delta_{A}\right)$.

Proof of Theorem 4.6. According to Theorem 2.2, the prime $P_{\tau}$ is associated to $\operatorname{in}_{w}\left(X_{A}^{*}\right)$ if and only if the polyhedral cone $w+\mathbb{R}_{>0}\left\{e_{\tau_{1}}, \ldots, e_{\tau_{c}}\right\}$ meets the tropicalization $\tau\left(X_{A}^{*}\right)$, which was described in Theorem 1.1 as $\mathcal{B}^{*}(A)+\operatorname{rowspace}(A)$.

The collection of cones $\mathbb{R}_{\geq 0} \sigma$ for $\sigma \in \mathcal{C}$ forms a unimodular triangulation of the co-Bergman fan $\mathcal{B}^{*}(A)$. This was proved by Ardila and Klivans [1, and we discussed it in Theorem 3.3. calling $\left(\mathbb{R}_{\geq 0} \sigma\right)_{\sigma \in \mathcal{C}}$ the flag fan $\mathcal{F}\left(\mathcal{L}_{M}\right)$ of the matroid given by the rows of a Gale dual $B$ of $A$. Therefore, (4.4) characterizes when $w+\mathbb{R}_{>0}\left\{e_{\tau_{1}}, \ldots, e_{\tau_{c}}\right\}$ meets $\mathbb{R}_{\geq 0} \sigma+\operatorname{rowspace}(A)$ for some $\sigma \in \mathcal{C}^{c}$. The multiplicity of this intersection is precisely the stated $n \times n$-determinant. This can be derived from Remarks 2.1 and 3.2 .

Our degree formula for the $A$-discriminant can now be rephrased in the following manner which is more conceptual and geometric.

Corollary 4.7. A monomial prime $P_{\tau}$ is associated to $\mathrm{in}_{w}\left(X_{A}^{*}\right)$ if and only if the cone $w+\mathbb{R}_{>0}\left\{e_{\tau_{1}}, \ldots, e_{\tau_{c}}\right\}$ meets the fan $\mathcal{B}^{*}(A)+\operatorname{rowspace}(A)$. The number of intersections, counted with multiplicity, is the multiplicity of $\operatorname{in}_{w}\left(X_{A}^{*}\right)$ along $P_{\tau}$.

\section{Computations, subdivisions, AND Singular tropical Hypersurfaces}

We start this section with a brief discussion of computational issues. The formula for the extremal terms of $\Delta_{A}$ in Theorem 1.2 gives rise to a practical method for computing the Newton polytope of the $A$-discriminant $\Delta_{A}$. Indeed, the co-Bergman 
fan of the matrix $A$ can be computed efficiently by gluing local Bergman fans, as explained in [10, Algorithm 5.5]. See Examples 5.7, 5.8 and 5.9 in [10] for some non-trivial computations. Extending the software used for those computations, we wrote a maple program for evaluating the formula (1.2) in Theorem 1.2. The input for our program consists of three positive integers $d, n, R$, and a $d \times n$-matrix $A$ which is assumed to be non-defective. The output is a list of initial monomials $\operatorname{in}_{w}\left(\Delta_{A}\right)$ of the $A$-discriminant $\Delta_{A}$, for $R$ randomly chosen vectors $w$ in $\mathbb{N}^{n}$. Our maple implementation is available upon request from any of the authors.

Note that in case $A$ is non-defective, it is possible to recover $\Delta_{A}$ up to constant from its Newton polytope $N\left(\Delta_{A}\right)$ by solving a linear system of equations. Namely, consider a generic polynomial $g$ with exponents in the lattice points of $N\left(\Delta_{A}\right)$. Imposing the condition that $g$ vanishes on the image of the rational parametrization of $\Delta_{A}$ given in Proposition 4.1 and Lemma 4.2 translates into a system of linear equations in the coefficients of $g$ whose solution space is 1-dimensional.

Example 5.1. Let $d=4$ and $n=8$, and consider the matrix $A$ below. The corresponding $A$-discriminant is the mixed discriminant of the two bivariate polynomials $f_{1}, f_{2}$ whose exponent vectors are read, respectively, from the first four and last four columns of the last two rows of the matrix. The non-vanishing of $\Delta_{A}$ expresses the condition that the intersection $f_{1}=f_{2}=0$ is transversal. What follows is the output of our maple program on this input. On a fast workstation, our code takes about half a second to compute the co-Bergman fan. Afterwards it takes about one second per initial monomial. So the total running time for this matrix is about $R$ seconds, where $R$ is the number of iterations specified by the user:

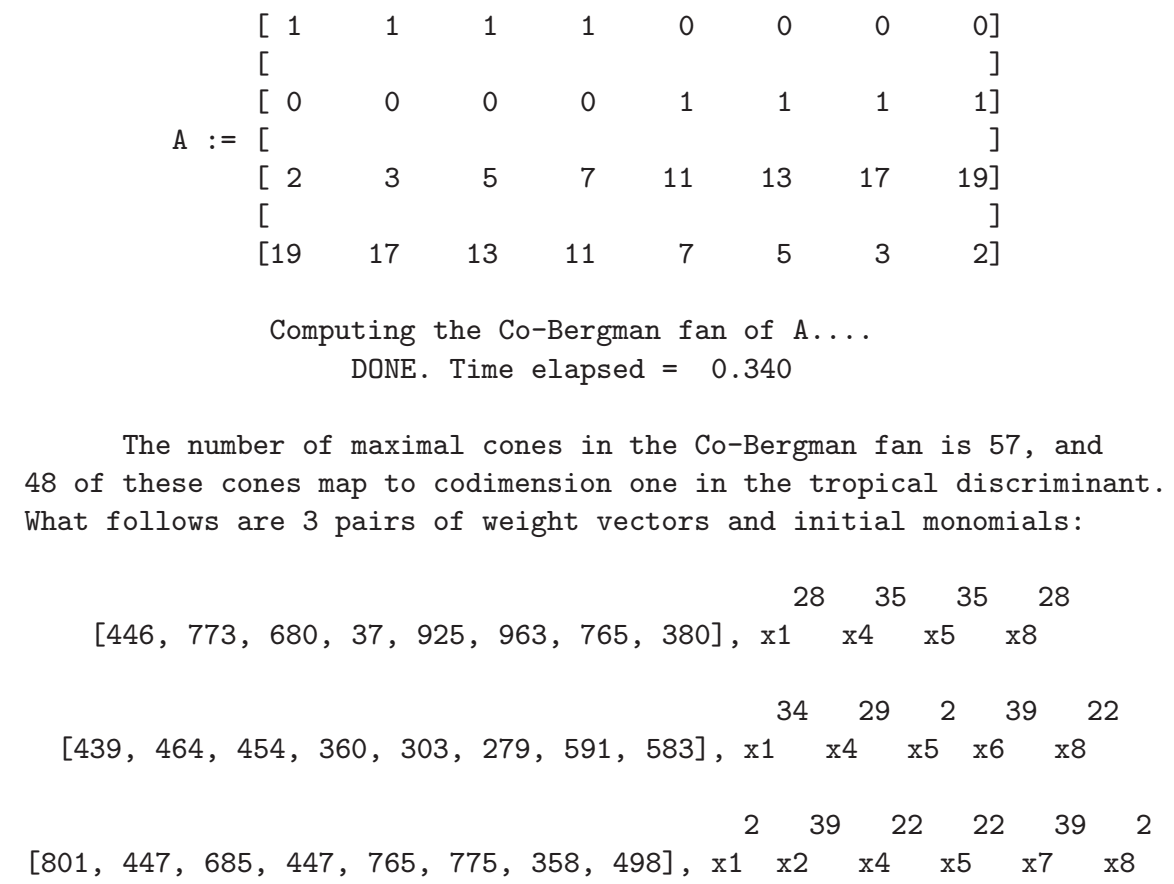

From this output we see that this $A$-discriminant is a polynomial of degree 126 . We discuss the geometric meaning of this example in Section 6. 
Gel'fand, Kapranov and Zelevinsky [13] established the relationship between the $A$-discriminant and the secondary fan of a point configuration. The secondary fan parametrizes the regular polyhedral subdivisions of $A$. It is shown in [13, that, in the non-defective case, the $A$-discriminant divides the principal $A$-determinant. Hence, the Newton polytope of $\Delta_{A}$ is a Minkowski summand of the secondary polytope of $A$, which in turn implies that the secondary fan $\Sigma(A)$ is a refinement of the normal fan of the Newton polytope of $\Delta_{A}$. The tropical discriminant $\tau\left(X_{A}^{*}\right)$ being the codimension 1-skeleton of the normal fan of the Newton polytope of $\Delta_{A}$, we obtain that $\tau\left(X_{A}^{*}\right)$ is a subfan of the secondary fan $\Sigma(A)$.

For a given $w \in \tau\left(X_{A}^{*}\right)$, the corresponding regular subdivision $\Pi_{w}$, i.e., the support cell in $\Sigma(A)$, is obtained as follows: Lift the point configuration $a_{1}, \ldots, a_{n}$ from $\mathbb{R}^{d}$ into $\mathbb{R}^{d+1}$ by extending with the coordinates of $w$ to $\left(a_{1}, w_{1}\right), \ldots,\left(a_{n}, w_{n}\right)$ in $\mathbb{R}^{d+1}$. The cells of $\Pi_{w}$ are the subsets of $A$ corresponding to the lower facets of the convex hull of the lifted point configuration.

In fact, we conjecture that membership in $\tau\left(X_{A}^{*}\right)$ depends only on the regular subdivision specified by the vector $w$, even in the defective case:

Conjecture 5.2. For any point configuration $A$, the tropical discriminant $\tau\left(X_{A}^{*}\right)$ is a union of cones in the secondary fan $\Sigma(A)$.

Another notion that arose in the work of Gel'fand, Kapranov and Zelevinsky is the notion of $\Delta$-equivalence of regular triangulations of a non-defective point configuration: Let $\Pi_{w}$ and $\Pi_{w^{\prime}}$ be two regular triangulations which are neighbors in the secondary fan $\Sigma(A)$. This means that their cones in $\Sigma(A)$ share a common face of codimension one. We say that $\Pi_{w}$ and $\Pi_{w^{\prime}}$ are $\Delta$-equivalent if they specify the same leading monomial of the $A$-discriminant, i.e., $\operatorname{in}_{w}\left(\Delta_{A}\right)=\operatorname{in}_{w^{\prime}}\left(\Delta_{A}\right)$. The $\Delta$-equivalence classes of regular triangulations of a point configuration $A$ define a partition of the set of maximal cones in the secondary fan $\Sigma(A)$.

Remark 5.3. The $\Delta$-equivalence classes are in bijection with the connected components of the complement $\mathbb{R}^{n} \backslash \tau\left(X_{A}^{*}\right)$ of the tropical discriminant.

We return to Example 4.4 to illustrate the relation of the tropical discriminant and the secondary fan of a point configuration as well as the notion of $\Delta$-equivalence.

Example 5.4. Let $A$ be the $3 \times 6$-matrix in Example 4.4 whose toric variety is the Veronese surface in $\mathbb{C P}^{5}$ and whose tropical discriminant $\tau\left(X_{A}^{*}\right)$ was depicted in Figure 2, We now identify $\tau\left(X_{A}^{*}\right)$ as a subfan of the secondary fan $\Sigma(A)$. Both of these 2-dimensional fans are drawn as planar graphs in Figure 3. The graph $\Sigma(A)$ is dual to the three-dimensional associahedron, and its 14 regions are labeled with the 14 regular triangulations of the configuration $A$. The tropical discriminant $\tau\left(X_{A}^{*}\right)$ is the subgraph which is indicated by solid lines. Edges of $\Sigma(A)$ that do not belong to $\tau\left(X_{A}^{*}\right)$ are dashed. The 14 regular triangulations of $A$ occur in five $\Delta$-equivalence classes corresponding to the open cells in the complement of $\tau\left(X_{A}^{*}\right)$.

The magnification on the left in Figure 3 shows a portion of the (dual) secondary polytope of $A$ with corresponding polyhedral subdivisions of the 6-point configuration indicated next to the faces.

We present two more examples which illustrate the various natural fan structures on the tropical discriminant. In the non-defective case, the fan structure inherited 


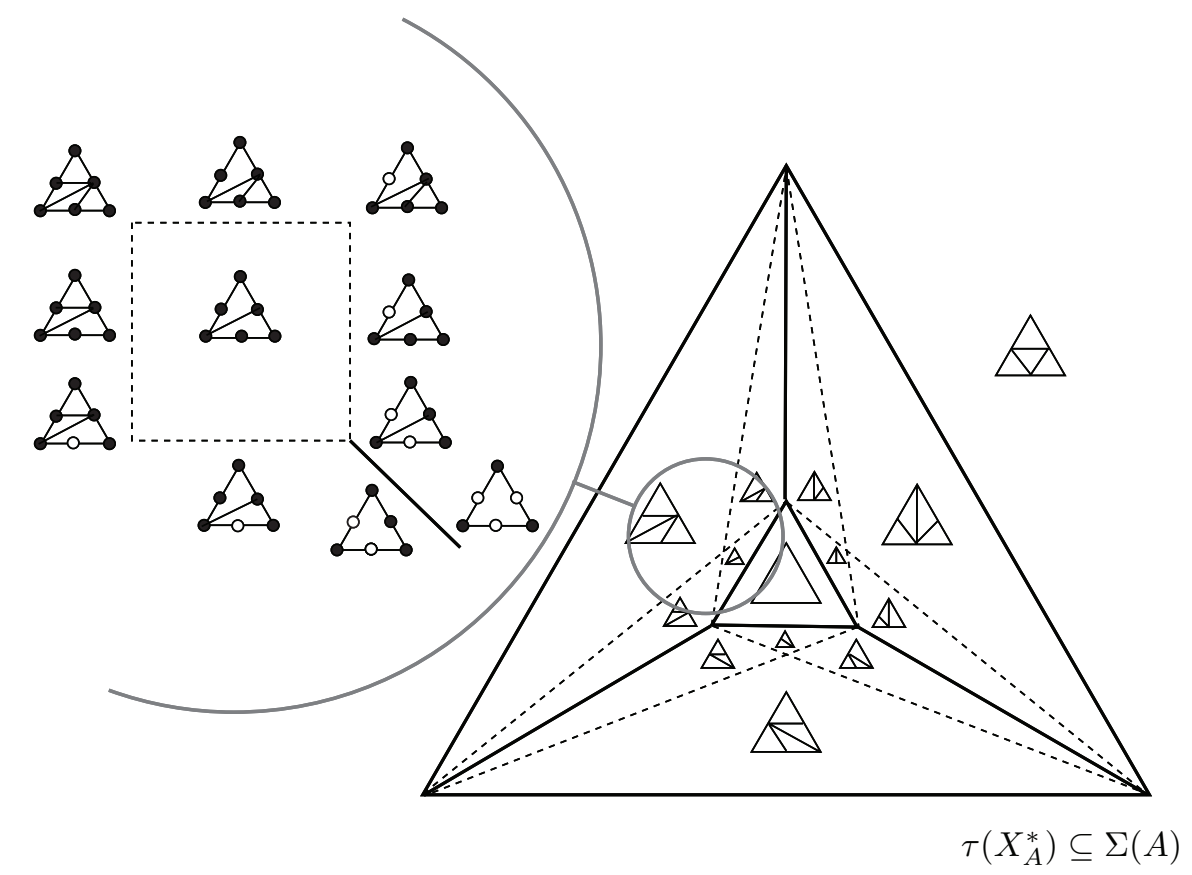

FIgURE 3. The tropical discriminant is a subfan of the secondary fan

from the secondary fan refines the fan structure dual to the Newton polytope of $\Delta_{A}$. The following example shows that a proper subdivision can occur.

Example 5.5. Let $d=3, n=6$, and consider the non-defective configuration

$$
A=\left(\begin{array}{llllll}
1 & 1 & 1 & 1 & 0 & 0 \\
0 & 0 & 0 & 0 & 1 & 1 \\
0 & 1 & 2 & 3 & 0 & 1
\end{array}\right) .
$$

Here, $\Delta_{A}=x_{1} x_{6}^{3}-x_{2} x_{5} x_{6}^{2}+x_{3} x_{5}^{2} x_{6}-x_{4} x_{5}^{3}$. Modulo the row space of $A$, the codimension 1-skeleton of the secondary fan is 2-dimensional. It is the cone over a planar graph with eight vertices and 18 edges. The tropical discriminant $\tau\left(X_{A}^{*}\right)$ corresponds to the induced subgraph on six of the vertices, namely, the images of the 6-dimensional weights $e_{1}, e_{2}, e_{3}, e_{4}, 2 e_{1}+e_{2}$ and $e_{3}+2 e_{4}$. Here, the secondary fan strictly refines the Gröbner fan on $\tau\left(X_{A}^{*}\right)$. The latter is combinatorially a complete graph $K_{4}$ with vertices $e_{1}, e_{2}, e_{3}$ and $e_{4}$, while the former has the edges $e_{1}, e_{2}$ and $e_{3}, e_{4}$ subdivided by the vertices $2 e_{1}+e_{2}$ and $e_{3}+2 e_{4}$, respectively.

For defective configurations, the Gröbner fan structure can properly refine the secondary fan structure on $\tau\left(X_{A}^{*}\right)$, as the following example shows.

Example 5.6. We take $d=4, n=9$, and consider the defective configuration

$$
A=\left(\begin{array}{lllllllll}
1 & 1 & 1 & 0 & 0 & 0 & 0 & 0 & 0 \\
0 & 0 & 0 & 1 & 1 & 1 & 0 & 0 & 0 \\
0 & 0 & 0 & 0 & 0 & 0 & 1 & 1 & 1 \\
0 & 1 & 2 & 0 & 1 & 2 & 0 & 1 & 2
\end{array}\right) .
$$


The tropical discriminant $\tau\left(X_{A}^{*}\right)$ is a 7 -dimensional fan in $\mathbb{R}^{9}$, regarded as a 6 dimensional polyhedral complex. Combinatorially, it is an immersion of the complete tripartite hypergraph $K_{3,3,3}$. The Gröbner fan subdivision has 51 maximal cones and it strictly refines the secondary fan subdivision which has only 49 cones. Indeed, the vector $w=(0,1,0,0,1,0,0,1,0)$ lies in the relative interior of a maximal cone of the secondary fan subdivision which breaks into three maximal cones in the Gröbner fan subdivision. This example was verified by applying the software Gfan [3] to the equations defining $X_{A}^{*}$. In particular, we found that $\operatorname{in}_{w}\left(X_{A}^{*}\right)$ is the codimension two primary ideal generated by the determinant of the $3 \times 3$-matrix

$$
\left(\begin{array}{lll}
x_{1} & x_{4} & x_{7} \\
x_{2} & x_{5} & x_{8} \\
x_{3} & x_{6} & x_{9}
\end{array}\right)
$$

plus the square of the ideal of $2 \times 2$-minors of the $2 \times 3$-matrix $\left(\begin{array}{lll}x_{1} & x_{4} & x_{7} \\ x_{3} & x_{6} & x_{9}\end{array}\right)$.

There is yet another way to interpret the $A$-discriminant and its tropicalization. For $A \in \mathbb{Z}^{d \times n}$, under the conditions imposed above, the $A$-discriminant is the closure of the set of points $\left[x_{1}: \cdots: x_{n}\right]$ in $\mathbb{C P}^{n-1}$ such that the hypersurface in $\left(\mathbb{C}^{*}\right)^{d}$ defined by the Laurent polynomial

$$
f(t)=\sum_{i=1}^{n} x_{i} t^{a_{i}}
$$

has a singular point in $\left(\mathbb{C}^{*}\right)^{d}$.

In the tropical world, for $w \in \mathbb{R}^{n}$, the regular polyhedral subdivision $\Pi_{w}$ of $A$ is geometrically dual to the tropical hypersurface defined by the tropical polynomial $\bigoplus_{i=1}^{n} w_{i} \odot t^{\odot a_{i}}$. Hence, we may consider such a tropical hypersurface to be singular whenever the vector $w$ lies in the tropical discriminant $\tau\left(X_{A}^{*}\right)$. In this sense, our object of study in this article is the space of singular tropical hypersurfaces and Theorem 1.2 gives a refined formula for the degree of that space.

Remark 5.7. The tropical discriminant $\tau\left(X_{A}^{*}\right)$ is the polyhedral space which parametrizes all singular tropical hypersurfaces with fixed monomial support $A$.

This relates our results to the celebrated work of Mikhalkin [18, on GromovWitten invariants. For $d=3$, the $A$-discriminant $X_{A}^{*}$ parametrizes singular curves on the toric surface $X_{A}$. Our formula for the degree of $X_{A}^{*}$ is consistent with the lattice paths count in [18] for the number of nodal curves on $X_{A}$ of genus $g-1$ through $n-2$ fixed points, where $g$ is the number of interior points in the lattice polytope $Q_{A}$. It would be interesting to explore possible applications of our combinatorial approach to Gromov-Witten theory. The work of Gathmann and Markwig [12] offers an algebraic setting for such a study (see also the recent preprint [17, which addressed this question after the first version of our paper).

\section{Cayley COnfigurations And Resultant varieties}

One of the main applications of $A$-discriminants is the study of resultants in elimination theory. The configurations $A$ which arise in elimination theory have a special combinatorial structure arising from the Cayley trick. See [13, §3.2.D] for a geometric introduction. Based on the results of the earlier sections, we here study 
the combinatorics and geometry of tropical resultants, and we generalize the positive degree formula for resultants in [22] to resultant varieties of arbitrary codimension.

Let $A_{1}, \ldots, A_{m}$ be finite subsets of $\mathbb{Z}^{r}$. Their Cayley configuration is defined as

$$
A=\left\{e_{1}\right\} \times A_{1} \cup\left\{e_{2}\right\} \times A_{2} \cup \cdots \cup\left\{e_{m}\right\} \times A_{m} \subset \mathbb{Z}^{m} \times \mathbb{Z}^{r},
$$

where $e_{1}, \ldots, e_{m}$ is the standard basis of $\mathbb{Z}^{m}$. To be consistent with our notation in Sections 1-5, we can regard $A$ as a $d \times n$-matrix with $d=m+r$ and $n=$ $\left|A_{1}\right|+\left|A_{2}\right|+\cdots+\left|A_{m}\right|$. As in [13, $\left.\S 8.1\right]$ and [22, the Cayley configuration $A$ represents the following system of $m$ Laurent polynomial equations in $r$ unknowns:

$$
\sum_{u \in A_{1}} x_{1, u} z^{u}=\sum_{u \in A_{2}} x_{2, u} z^{u}=\cdots=\sum_{u \in A_{m}} x_{m, u} z^{u}=0
$$

Here $z=\left(z_{1}, \ldots, z_{r}\right)$ are coordinates on $\left(\mathbb{C}^{*}\right)^{r}$ and we use multi-index notation $z^{u}=z_{1}^{u_{1}} \cdots z_{r}^{u_{r}}$. Our earlier examples include the following Cayley configurations:

- In Example 5.1 we have $m=2, r=2$, and the system (6.2) takes the form

$$
\begin{gathered}
x_{1} z_{1}^{2} z_{2}^{19}+x_{2} z_{1}^{3} z_{2}^{17}+x_{3} z_{1}^{5} z_{2}^{13}+x_{4} z_{1}^{7} z_{2}^{11}=0, \\
x_{5} z_{1}^{11} z_{2}^{7}+x_{6} z_{1}^{13} z_{2}^{5}+x_{7} z_{1}^{17} z_{2}^{3}+x_{8} z_{1}^{19} z_{2}^{2}=0 .
\end{gathered}
$$

- In Example 5.5 we have $m=2, r=1, A_{1}=\{0,1,2,3\}$ and $A_{2}=\{0,1\}$. The $A$-discriminant is the Sylvester resultant

$$
\Delta_{A}=\operatorname{det}\left(\begin{array}{cccc}
x_{1} & x_{2} & x_{3} & x_{4} \\
x_{5} & x_{6} & 0 & 0 \\
0 & x_{5} & x_{6} & 0 \\
0 & 0 & x_{5} & x_{6}
\end{array}\right) .
$$

- In Example 5.6 we have $m=3, r=1$ and $A_{1}=A_{2}=A_{3}=\{0,1,2\}$, and the system (6.2) consists of three quadratic equations in one unknown $z$ :

$$
x_{1}+x_{2} z+x_{3} z^{2}=x_{4}+x_{5} z+x_{6} z^{2}=x_{7}+x_{8} z+x_{9} z^{2}=0 .
$$

The variety $X_{A}^{*}$ of all solvable systems of this form has codimension two.

Returning to the general case, we say that the Cayley configuration $A$ is essential if the Minkowski sum $\sum_{i \in I} A_{i}$ has affine dimension at least $|I|$ for every subset $I$ of $\{1, \ldots, m\}$ with $|I| \leq r$. The resultant variety of the Cayley configuration $A$ is the Zariski closure in $\mathbb{C P}^{n-1}$ of the set of all points $\left(x_{1}: x_{2}: \ldots: x_{n}\right)$ whose corresponding system (6.2) has a solution $z$ in $\left(\mathbb{C}^{*}\right)^{r}$. The following result is a generalization of Proposition 1.7 in [13, §9.1.A] and of Proposition 5.1 in [4].

Proposition 6.1. The resultant variety of any Cayley configuration $A$ contains the A-discriminant $X_{A}^{*}$. If $m \geq r+1$ and the Cayley configuration $A$ is essential, then the resultant variety and the A-discriminant coincide.

Proof. Consider the hypersurface in $\left(\mathbb{C}^{*}\right)^{m} \times\left(\mathbb{C}^{*}\right)^{r}$ defined by the equation

$$
\sum_{i=1}^{m} \sum_{u \in A_{i}} x_{i, u} \cdot t_{i} z^{u}=0 .
$$

If $(t, z) \in\left(\mathbb{C}^{*}\right)^{m+r}$ is a singular point on this hypersurface, then $z \in\left(\mathbb{C}^{*}\right)^{r}$ is a solution to (6.2). This proves the inclusion. If $A$ is essential, a linear algebra argument as in [13, $§ 9.1$.A] shows that every solution $z$ of $(\underline{6.2})$ arises in this way. 
The hypothesis that $A$ be essential is necessary for the equality of the resultant variety and the $A$-discriminant, even when $m=r+1$, the situation of classical elimination theory. The following simple example illustrates the general behavior.

Example 6.2. Let $r=2, m=3, A_{1}=A_{2}=\{(0,0),(1,0)\}$, and $A_{3}=\{(0,0),(1,0)$, $(0,1),(1,1)\}$. The Cayley matrix $A$ represents a toric 4 -fold $X_{A}$ in $\mathbb{C P}^{7}$. It is not essential since $A_{1}+A_{2}$ is 1 -dimensional. The system (6.2) equals

$$
x_{1}+x_{2} z_{1}=x_{3}+x_{4} z_{1}=x_{5}+x_{6} z_{1}+x_{7} z_{2}+x_{8} z_{1} z_{2}=0 .
$$

The resultant variety has codimension one, with equation $x_{1} x_{4}=x_{2} x_{3}$, but the $A$-discriminant $X_{A}^{*}$ has codimension three. In fact, we have $X_{A}^{*}=X_{A}$ in this case.

For the rest of this section we consider an essential Cayley configuration as in (6.1) with $m \geq r+1$ blocks and we set $c=m-r$. Then the following result holds.

Lemma 6.3. The resultant variety $X_{A}^{*}$ has codimension $c$.

Proof. Let $W$ denote the incidence variety consisting of all pairs $(x, z)$ in $\mathbb{C P}^{n-1} \times$ $\left(\mathbb{C}^{*}\right)^{r}$ such that (6.2) holds. Let $\pi_{1}: W \rightarrow \mathbb{C P}^{n-1}$ be the projection to the first factor. By Proposition 6.1 the resultant variety $X_{A}^{*}$ coincides with the closure of $\pi_{1}(W)$. Looking at the second projection $\pi_{2}: W \rightarrow\left(\mathbb{C}^{*}\right)^{r}$, which is surjective and whose fibers are linear spaces of dimension $n-1-m$, we deduce that $W$ is irreducible and has dimension $(n-1-m)+r=n-1-c$. Then, $\operatorname{dim}\left(X_{A}^{*}\right) \leq n-1-c$.

Given a generic choice of coefficients $x_{i}$ for the first $r$ polynomials, it follows from the essential hypothesis and Bernstein's Theorem that the first $r$ equations in (6.2) have a common solution $z \in\left(\mathbb{C}^{*}\right)^{r}$. We can freely choose all but one of the coefficients of the last $c$ polynomials so that $z$ solves (6.2). This implies that $\operatorname{dim}\left(X_{A}^{*}\right) \geq n-1-c$, and the lemma follows.

Corollary 4.5 asserts that there exists a chain $\sigma_{1}, \ldots, \sigma_{n-2 m}$ of $(0,1)$-vectors representing the supports of vectors in the kernel of $A$ such that the rank of the matrix $\left(A^{t}, \sigma_{1}, \ldots, \sigma_{n-2 m}\right)$ is precisely $n-c$. We present an explicit way of choosing such a chain. By performing row operations, we can assume that each set $A_{i}$ contains the origin. Set $B_{i}=A_{i} \backslash\{0\}$. Let $b_{i} \in B_{i}$ for $i=1, \ldots, r$ such that $b_{1}, \ldots, b_{r}$ are linearly independent. Such elements exist because the family of supports is essential. Now, for any other element $a$ in $B=\left(B_{1} \cup \cdots \cup B_{r+c}\right) \backslash\left\{b_{1}, \ldots, b_{r}\right\}$, we can find an element $v_{a}$ in $\operatorname{ker}(A)$ with support corresponding to the origin in each $A_{i}$ for $i$ from 1 to $r$, union the variables corresponding to $b_{1}, \ldots, b_{r}$ and $a \in B_{j}$, plus the origin of $A_{j}$ in case $j>r$. Choose any such $a \in B_{r+1}$ and let $\sigma_{1}$ be the support of $v_{a}$; it will have $2 r+2$ non-zero coordinates. Add a new point $a^{\prime}$ in $B$. We can assume that the support of $v_{a}+v_{a}^{\prime}$ equals the union of their supports. Let $\sigma_{2}$ be the associated support vector. We continue in this manner, adding a new point in $B$ at a time and considering a new element in the chain of support vectors, but avoiding to pick all of $B_{1} \cup \cdots \cup B_{r+1}$ and all of each of $B_{r+2}, \ldots, B_{r+c}$. This produces precisely

$1+\left(n_{1}-2+\cdots+n_{r+1}-2\right)-1+\left(n_{r+2}-2\right)+\cdots+\left(n_{r+c}-2\right)=n-2 r-2 c=n-2 m$ vectors $\sigma_{1}, \ldots, \sigma_{n-2 m}$ in $\mathcal{C}(A)$. It is straightforward to check that the rank of the submatrix of $\left(A^{t}, \sigma_{1}, \ldots, \sigma_{n-2 m}\right)$ given by the first $m$ and the last $n-2 m$ columns has maximal rank $n-m$. Note that this is just a $(0,1)$ matrix. Adding the last $r$ columns of $A^{t}$ containing the information about the specific supports $A_{1}, \ldots, A_{m}$ 
will increase the rank by $r$, as a consequence of the fact that the family is essential. Therefore, the rank of the matrix $\left(A^{t}, \sigma_{1}, \ldots, \sigma_{n-2 m}\right)$ is precisely $n-m+r=n-c$.

We identify $\{1,2, \ldots, n\}$ with the disjoint union of the sets $A_{1}, A_{2}, \ldots, A_{m}$. Thus a generic vector $w \in \mathbb{R}^{n}$ assigns a height to each point in any of the $A_{i}$, and it defines a tight coherent mixed subdivision $\Delta_{w}$ of the Minkowski sum $\sum_{i=1}^{m} A_{i}$ (cf. [22]). When $c=1$, the initial form with respect to $w$ of the mixed resultant is described in [22, Theorem 2.1] in terms of the sum of volumes of suitable mixed cells of the tight coherent mixed decomposition (TCMD) induced by $w$. We next generalize this result to resultant varieties of arbitrary codimension $c$. For a classical study of resultant ideals of dense homogeneous polynomials we refer to [14].

Theorem 6.4. A codimension c monomial prime $P_{\tau}=\left\langle x_{\tau_{1}}, \ldots, x_{\tau_{c}}\right\rangle$ is a minimal prime of the monomial ideal $\operatorname{in}_{w}\left(X_{A}^{*}\right)$ only if $\tau$ consists of one point each from $c$ of the $A_{j}$. The multiplicity of $P_{\tau}$ is the total volume of all mixed cells in the tight coherent mixed subdivision $\Delta_{w}$ which use the points of $\tau$ in their decomposition.

Proof. The resultant variety $X_{A}^{*}$ is irreducible (by Proposition 6.1), and it has codimension $c$ (by Lemma [6.3). This implies (by [15, Theorem 1]) that every minimal prime of the initial monomial ideal $\operatorname{in}_{w}\left(X_{A}^{*}\right)$ has codimension $c$. Let $P_{\tau}=\left\langle x_{\tau_{1}}, \ldots, x_{\tau_{c}}\right\rangle$ be such a minimal prime. After relabeling, we may assume that each $x_{\tau_{i}}$ in $P_{\tau}$ is a coefficient of one of the last $c$ Laurent polynomials in (6.2).

The proof for the case $c=1$ is given in [22, §2], and the proof for $c>1$ uses the same general technique. We write $f_{i}$ for the $i$ th Laurent polynomial in (6.2), but with $x_{i}$ replaced by $x_{i} \epsilon^{w_{i}}$. Let $\mathbf{K}$ be the algebraic closure of the field of rational functions over $\mathbb{C}\{\{\epsilon\}\}$ in the coefficients of the first $r$ Laurent polynomials $f_{1}, \ldots, f_{r}$, let $\mathbf{x}$ denote the vector of all coefficients of the last $c$ Laurent polynomials $f_{r+1}, \ldots, f_{m}$, and consider the polynomial ring $\mathbf{K}[\mathbf{x}]$. Let $\mu$ denote the mixed volume of the Newton polytopes of the polynomials $f_{1}, \ldots, f_{r}$. Then, by Bernstein's Theorem the system $f_{1}=\cdots=f_{r}=0$ has $\mu$ distinct roots $\mathbf{z}_{1}(\epsilon), \ldots, \mathbf{z}_{\mu}(\epsilon)$ in $\left(\mathbf{K}^{*}\right)^{r}$.

For any $j \in\{1,2, \ldots, \mu\}$, the ideal $I_{j}=\left\langle f_{r+1}\left(\mathbf{z}_{i}(\epsilon)\right), \ldots, f_{m}\left(\mathbf{z}_{i}(\epsilon)\right)\right\rangle$ is generated by linear forms in $\mathbf{K}[\mathbf{x}]$. The intersection of these ideals, $I=I_{1} \cap I_{2} \cap \cdots \cap I_{\mu}$, is an ideal of codimension $c$ and degree $\mu$ in $\mathbf{K}[\mathbf{x}]$. Geometrically, we obtain $I$ by embedding the prime ideal of $X_{A}^{*}$ into $\mathbf{K}[\mathbf{x}]$ and then replacing $x_{i}$ by $x_{i} \epsilon^{w_{i}}$. This is the higher codimension version of the product formula for resultants 22, equation (14)].

The ideal $I$ represents a flat family, and its special fiber $\left.I\right|_{\epsilon=0}$ at $\epsilon=0$ coincides with the special fiber of the image of $\operatorname{in}_{w}\left(X_{A}^{*}\right)$ in $\mathbf{K}[\mathbf{x}]$. In particular, $P_{\tau}$ is an associated prime of $\left.I\right|_{\epsilon=0}$, and it contains one of the ideals $\left.I_{j}\right|_{\epsilon=0}$. Since the generators of $I_{j}$ are $c$ linear forms in disjoint groups of unknowns $x_{\ell}$, we see that $P_{\tau}$ contains one unknown from each group. This proves the first statement in Theorem 6.4

After relabeling, we may assume that $x_{\tau_{j}}$ is a coefficient of $f_{r+j}$ for $j=1, \ldots, c$. Each root $\mathbf{z}_{j}(\epsilon)$ corresponds to a mixed cell $C$ in the TCMD of the small Minkowski sum $A_{1}+\cdots+A_{r}$ defined by the restriction of $w$. By the genericity of $w$, the mixed cell $C$ corresponds to a unique cell $C^{\prime}$ in the TCMD $\Delta_{w}$ of the big Minkowski sum $A_{1}+\cdots+A_{r}+A_{r+1}+\cdots+A_{m}$, and every mixed cell of $\Delta_{w}$ arises in this manner. The reasoning above implies that the mixed cell $C^{\prime}$ uses the points of $\tau$ in its decomposition if and only if $\left.I_{j}\right|_{\epsilon=0}=P_{\tau}$ in $\mathbf{K}[\mathbf{x}]$. This completes the proof.

The first assertion in Theorem 6.4 can also be derived more easily, namely, from the fact that for any $(r+1)$-element subset $I$ of $\{1, \ldots, m\}$, the mixed resultant 
of the configurations $A_{i}, i \in I$, vanishes on $X_{A}^{*}$ and only contains unknowns $x_{i, a}$ with $i \in I$. However, for the multiplicity count in the second assertion we need the "product formula" developed above. Theorem 6.4 has the following corollary.

Corollary 6.5. The degree of the resultant variety $X_{A}^{*}$ is the sum of the mixed volumes $M V\left(A_{i_{1}}, \ldots, A_{i_{r}}\right)$ as $\left\{i_{1}, \ldots, i_{r}\right\}$ runs over all $r$-element subsets of $\{1, \ldots, n\}$.

We present two examples to illustrate Theorem 6.4 and Corollary 6.5.

Example 6.6. Let $m=3, r=1$ and $A_{1}=A_{2}=A_{3}=\{0,1,2\}$ as in Example 5.6. and choose $w \in \mathbb{R}^{9}$ which represents the reverse lexicographic term order. Then

$$
\operatorname{in}_{w}\left(X_{A}^{*}\right)=\left\langle x_{3} x_{5} x_{7}, x_{6}^{2} x_{7}^{2}, x_{3} x_{6} x_{7}^{2}, x_{3}^{2} x_{7}^{2}, x_{3} x_{4} x_{6} x_{7}, x_{3}^{2} x_{4} x_{7}, x_{3}^{2} x_{4}^{2}, x_{2} x_{4} x_{6}^{2} x_{7}\right\rangle .
$$

This ideal has seven associated primes, of which three are minimal: $\left\langle x_{3}, x_{6}\right\rangle$, $\left\langle x_{3}, x_{7}\right\rangle$, and $\left\langle x_{4}, x_{7}\right\rangle$. They correspond to the three mixed cells $(3,6,\{7,8,9\})$, $(3,\{4,5,6\}, 7)$ and $(\{1,2,3\}, 4,7)$ of the TCMD $\Delta_{w}$ of $A_{1}+A_{2}+A_{3}=\{0,1, \ldots, 6\}$. Each mixed cell has volume two, which implies that the degree of $X_{A}^{*}$ is $2+2+2=6$.

Example 6.7. Let $r=2$ and $m=4$ and take the $A_{i}$ to be the four subtriangles of the square with vertices $(0,0),(0,1),(1,0)$ and $(1,1)$. Here (6.2) is a system of four equations in two unknowns $z_{1}$ and $z_{2}$ which can be written in matrix form as

$$
\left(\begin{array}{cccc}
x_{1} & x_{2} & x_{3} & 0 \\
x_{4} & x_{5} & 0 & x_{6} \\
x_{7} & 0 & x_{8} & x_{9} \\
0 & x_{10} & x_{11} & x_{12}
\end{array}\right) \cdot\left(\begin{array}{c}
1 \\
z_{1} \\
z_{2} \\
z_{1} z_{2}
\end{array}\right)=\left(\begin{array}{l}
0 \\
0 \\
0 \\
0
\end{array}\right) .
$$

The resultant variety $X_{A}^{*} \subset \mathbb{C P}^{11}$ has codimension 2 and degree $12=\left(\begin{array}{l}4 \\ 2\end{array}\right) \cdot 2$. This is the sum over the mixed areas of the $\left(\begin{array}{l}4 \\ 2\end{array}\right)$ pairs of triangles. The mixed area is 2 for each such pair. Using computer algebra, we find that the prime ideal of the resultant variety $X_{A}^{*}$ is generated by the $4 \times 4$ determinant in (6.3) together with ten additional polynomials of degree six in the $x_{i}$. Theorem 6.4 gives a combinatorial recipe for constructing all the initial monomial ideals of this prime ideal.

We conclude with a brief discussion of the tropical resultant $\tau\left(X_{A}^{*}\right)$. The results in Section 5 characterize this polyhedral fan in terms of regular subdivisions of $A$, and we will now rephrase this characterization in terms of coherent mixed subdivisions of $\left(A_{1}, \ldots, A_{m}\right)$. Theorem 5.1 in 22 implies that every regular subdivision $\Pi_{w}$ of $A$ corresponds uniquely to a coherent mixed subdivision (CMD), which we denote by $\Delta_{w}$. Note that $\Pi_{w}$ is a polyhedral cell complex of dimension $r+m-1$ while $\Delta_{w}$ has only dimension $r$, and $\Pi_{w}$ is a triangulation if and only if $\Delta_{w}$ is a TCMD.

Every cell $F$ of a CMD $\Delta_{w}$ decomposes uniquely as a Minkowski sum $F=$ $F_{1}+\cdots+F_{m}$, where $F_{i} \subset A_{i}$ for all $i$. We write $\bar{F}$ for the corresponding cell of $\Delta_{w}$. We say that the cell $F$ is fully mixed if each $F_{i}$ has affine dimension at least one. Using the techniques in the proof of Theorem 6.4 we can derive the following:

Proposition 6.8. The tropical resultant $\tau\left(X_{A}^{*}\right)$ equals the set

$$
\left\{w \in \mathbb{R}^{n}: \Delta_{w} \text { has a maximal cell } F \text { which is fully mixed }\right\} .
$$

Specializing to the classical case $c=1$, when $X_{A}^{*}$ is the hypersurface defined by the mixed resultant, we can now recover the combinatorial results in [22, §5]. In particular, the positive formula for the extreme monomials of the sparse resultant given in [22, Theorem 2.1] can now be recovered as a special case of Theorem 1.2 
Thus, one way to look at Sections 1-5 in the present paper is that these extend all results in 22. from essential Cayley configurations with $m=r+1$ to arbitrary configurations $A$. A perspective on how this relates to the approach of [13] is given by the points (a), (b), (c), (d) found at the end of the introduction in 22, pp. 208-209].

\section{ACKNOWLEDGEMENTS}

We thank the Forschungsinstitut für Mathematik at ETH Zürich for hosting

Alicia Dickenstein and Bernd Sturmfels in the summer of 2005. We are grateful to Jenia Tevelev and Josephine Yu for comments on this paper.

\section{REFERENCES}

1. F. Ardila, C. Klivans: The Bergman complex of a matroid and phylogenetic trees; Journal of Combinatorial Theory Series B 96 (2006), 38-49. MR2185977(2006i:05034)

2. R. Bieri, J. Groves: The geometry of the set of characters induced by valuations; Journal für die reine und angewandte Mathematik 347 (1984), 168-195. MR733052 (86c:14001)

3. T. Bogart, A. Jensen, D. Speyer, B. Sturmfels, R. Thomas: Computing tropical varieties; Journal of Symbolic Computation 42, Issue 1-2 (special issue on the occasion of MEGA 2005) (2007), 54-73. MR2284285

4. E. Cattani, A. Dickenstein, B. Sturmfels: Rational hypergeometric functions; Compositio Mathematica 128 (2001), 217-240. MR 1850183 (2003f:33016)

5. C. De Concini, C. Procesi: Hyperplane arrangements and holonomy equations; Selecta Mathematica (N.S.) 1 (1995), 495-535. MR1366623 (97k:14014)

6. A. Dickenstein, B. Sturmfels: Elimination theory in codimension 2; Journal of Symbolic Computation 34 (2002), 119-135. MR1930829(2003h:14073)

7. M. Einsiedler, M. Kapranov, D. Lind: Non-archimedean amoebas and tropical varieties; Journal für die reine und angewandte Mathematik 601 (2006), 139-157. MR2289207

8. E.M. Feichtner, D. Kozlov: Incidence combinatorics of resolutions; Selecta Mathematica (N.S.) 10 (2004), 37-60. MR2061222 (2006k:06008)

9. E.M. Feichtner, I. Müller: On the topology of nested set complexes; Proceedings of the American Mathematical Society 133 (2005), 999-1006. MR2117200(2006c:06005)

10. E.M. Feichtner, B. Sturmfels: Matroid polytopes, nested sets and Bergman fans; Portugaliae Mathematica (N.S.) 62 (2005), 437-468. MR2191630 (2006j:05036)

11. E.M. Feichtner, S. Yuzvinsky: Chow rings of toric varieties defined by atomic lattices; Inventiones Mathematicae 155 (2004), 515-536. MR2038195 (2004k:14009)

12. A. Gathmann, H. Markwig: The numbers of tropical plane curves through points in general position; Journal für die reine und angewandte Mathematik, to appear, math.AG/0504390.

13. I.M. Gel'fand, M.M. Kapranov, A.V. Zelevinsky: Discriminants, Resultants, and Multidimensional Determinants; Birkhäuser, Boston, MA, 1994. MR1264417 (95e:14045)

14. J.P. Jouanolou: Ideaux Résultants; Advances in Mathematics 37 (1980), 212-238. MR591727 (82c:14038)

15. M. Kalkbrener, B. Sturmfels: Initial complexes of prime ideals; Advances in Mathematics 116 (1995), 365-376. MR1363769 (97g:13043)

16. M. Kapranov: A characterization of A-discriminantal hypersurfaces in terms of the logarithmic Gauss map; Mathematische Annalen 290 (1991), 277-285. MR 1109634 (92j:14066)

17. E. Katz: The tropical degree of cones in the secondary fan, math.AG/ 0604290.

18. G. Mikhalkin: Enumerative tropical algebraic geometry in $\mathbb{R}^{2}$; Journal of the American Mathematical Society 18 (2005), 313-377. MR2137980(2006b:14097)

19. J. Richter-Gebert, B. Sturmfels, T. Theobald: First steps in tropical geometry; in: "Idempotent Mathematics and Mathematical Physics," Proceedings Vienna 2003 (G.L. Litvinov and V.P. Maslov, eds.), American Math. Society, Contemporary Mathematics 377 (2005), pp. 289-317. MR2149011(2006d:14073)

20. D. Speyer: Tropical Geometry; Ph.D. Dissertation, University of California at Berkeley, 2005.

21. D. Speyer, B. Sturmfels: The tropical Grassmannian; Advances in Geometry 4 (2004), 389411. MR2071813 (2005d:14089) 
22. B. Sturmfels: On the Newton polytope of the resultant; Journal of Algebraic Combinatorics 3 (1994), 207-236. MR 1268576 (95j:52024)

23. B. Sturmfels: Solving Systems of Polynomial Equations; CBMS Regional Conference Series in Mathematics 97, American Mathematical Society, Providence, 2002. MR1925796 (2003i:13037)

24. E. Tevelev: Compactifications of subvarieties of tori; American Journal of Mathematics, to appear, math.AG/0412329.

Departamento de Matemática, FCen, Universidad de Buenos Aires, (1428) B. Aires, Argentina

E-mail address: alidick@dm.uba.ar

Department of Mathematics, ETH Zürich, 8092 ZÜrich, Switzerland

Current address: Department of Mathematics, University of Stuttgart, 70569 Stuttgart, Germany

E-mail address: feichtne@igt.uni-stuttgart.de

Department of Mathematics, University of California, Berkeley, California 94720

E-mail address: bernd@math.berkeley.edu 